\title{
An Efficient Beam-Column Formulation for 3D Reinforced Concrete Frames
}

\author{
B.A. Izzuddin ${ }^{1}$, A.A.F.M. Siyam² and D. Lloyd Smith ${ }^{3}$
}

\begin{abstract}
This paper presents a new beam-column formulation which can be used for the accurate, yet efficient, modelling of 3D reinforced concrete $(\mathrm{R} / \mathrm{C})$ frames. The formulation is intended for modelling the nonlinear elastic behaviour of a whole $\mathrm{R} / \mathrm{C}$ beam-column with only one element, which is an essential ingredient of adaptive elasto-plastic analysis. On the longitudinal axis level, quartic shape functions are used to represent the two transverse displacements. A constant axial force criterion is employed instead of shape functions for the axial displacement, which is largely responsible for the accuracy of the proposed formulation. For concrete, the formulation assumes a nonlinear compressive stress-strain relationship and no tensile resistance; whereas for steel, a linear stress-strain relationship is utilised. On the crosssectional level, the formulation is capable of modelling the interaction between the axial force and the biaxial moments for a general $\mathrm{R} / \mathrm{C}$ cross-section, with explicit expressions obtained using a novel approach based on integration over triangular subdomains. The paper provides the details of the proposed formulation, and presents several verification examples to demonstrate the accuracy of this formulation and its ability to model the nonlinear elastic response of reinforced concrete beam-columns with only one element per member.
\end{abstract}

\footnotetext{
${ }^{1}$ Reader in Computational Structural Mechanics, Dept. of Civil \& Env. Eng'g, Imperial College, London.

${ }^{2}$ Research Student, Dept. of Civil \& Env. Eng'g, Imperial College, London.

${ }^{3}$ Reader in Structural Mechanics, Dept. of Civil \& Env. Eng'g, Imperial College, London.
} 


\section{KEYWORDS}

Reinforced concrete; Beam-columns; Nonlinear analysis; Efficient formulation.

\section{INTRODUCTION}

The need to predict the nonlinear response of structures, up to and beyond failure of individual components, has been the main motivation behind the development of new nonlinear structural analysis methods over the past few decades. For reinforced concrete $(\mathrm{R} / \mathrm{C})$ structures, the emergence of geometrically nonlinear finite element formulations has been accompanied by the development of advanced constitutive models for concrete and its interaction with steel reinforcement (Vidosa et al., 1991; Chen and Saleeb, 1994). However, although two- and three-dimensional finite element formulations can provide an accurate representation of the nonlinear response of $\mathrm{R} / \mathrm{C}$ members, they pose excessive computational demands which prevent their application to realistic structural assemblages. Consequently, one-dimensional formulations have been widely recognised as the most realistic alternative approach for the nonlinear response prediction of $\mathrm{R} / \mathrm{C}$ framed structures, combining modelling benefits, computational efficiency and reasonable accuracy.

The conventional one-dimensional modelling approach is based on using a considerable number of elasto-plastic elements for each member of the structure, so that the nonlinear response of the member is represented adequately. This approach can be prohibitively expensive, since it requires the use of computationally expensive elasto-plastic elements in all parts of the structure and from the start of analysis. Adaptive nonlinear analysis has recently emerged as the most effective approach for dealing with such computational inefficiencies, achieving computational savings of at least $75 \%$ in comparison with conventional nonlinear analysis of 3D steel frames (Izzuddin \& Elnashai, 1993-a) and 2D R/C frames (Karayannis et al., 1994). This superiority is attained by adaptive analysis through employing one elastic element per member at the start of analysis, and applying automatic mesh refinement into elasto-plastic elements only when and where necessary, during analysis and within the 
structure, respectively. Central to this concept of adaptive analysis is the availability of an accurate and simultaneously efficient elastic formulation which is capable of modelling the geometrically nonlinear elastic response of a whole member using only one element.

In formulating a nonlinear one-dimensional element, the two main considerations are i) the determination of the cross-section response and ii) the integration of the cross-section response over the element length to obtain the element response in terms of available element freedoms.

With regard to the first consideration, three main approaches have been widely adopted to determine the influence of concrete nonlinearity on the cross-section response. The first approach provides explicit expressions for the cross-section response parameters but has been restricted mainly to 2D frame analysis. Furthermore, most expressions have been provided for rectangular cross-sections (Rasheed \& Dinno, 1994), although a superposition technique applicable to general nonlinear elastic cross-sections was proposed by Izzuddin et al. (1994). The second approach employs interaction relationships on the cross-sectional level, based mainly on principles of plasticity (Sfakianakis \& Fardis, 1991), but has also been restricted to rectangular cross-sections. The third approach, commonly known as the fibre approach, is applicable to cross-sections of various shapes and materials, and is based on discretising the cross-section into a large number of small areas (Izzuddin \& Elnashai, 1993-a; Spacone \& Fillipou, 1994; El-Metwally et al., 1990) over which material stresses and strains are monitored. The latter approach is clearly more general; it is directly applicable to 3D frames, general cross-sections and elasto-plastic analysis. However, its computational requirements are evidently excessive in comparison with the former explicit approach, and cannot be justified for cross-sections which remain elastic. One aim of this work is to extend the explicit approach of Izzuddin et al. (1994) to model general elastic R/C cross-sections for 3D frame analysis.

With regard to the second consideration, displacement based finite element concepts are widely utilised, where polynomial shape functions are assumed for the transverse and axial 
centroidal displacements along the element length. Depending on the order of polynomials used, the resulting formulations can exhibit an over-stiff response, particularly for $\mathrm{R} / \mathrm{C}$ members which are subject to tensile cracking. This is mainly attributed to the violation of equilibrium along the element length when the displacement fields are prescribed to vary according to low order shape functions. Spacone et al. (1996) proposed a flexibility-based approach which enforces equilibrium along the element, but which satisfies displacement compatibility and the constitutive section response approximately to within a specified tolerance. However, this approach cannot readily incorporate geometric nonlinearities in the local element system; it requires a flexibility formulation for the cross-section response which may not be available if the cross-section is on a failure surface; and it poses considerable computational demands associated with the calculation and storage of the flexibility matrices of the various cross-sections. A hybrid elastic beam-column element for $2 \mathrm{D} \mathrm{R} / \mathrm{C}$ frames was proposed by Izzuddin et al. (1994), where quartic shape functions are used for the transverse displacements, and axial equilibrium is enforced over the element length, thus avoiding the use of shape functions for the axial displacement. This formulation was shown to provide accurate and efficient geometrically nonlinear elastic analysis of 2D R/C frames (Izzuddin et al., 1994; Karayannis et al., 1994), thus asserting its suitability for nonlinear adaptive analysis. Therefore, another aim of this work is to extend the aforementioned formulation to elastic beam-columns featuring in 3D R/C frames.

As mentioned above, this paper aims at extending a previous elastic $\mathrm{R} / \mathrm{C}$ beam-column formulation for 2D frames (Izzuddin et al., 1994) to 3D frame analysis. The paper proceeds with providing the details of nonlinear cross-section modelling, where general R/C crosssections are discretised into coarse rectangular areas, and a novel approach based on integration over triangular subdomains is employed. This enables the interaction between the biaxial bending moments and the axial force to be represented explicitly and accurately for a general elastic R/C cross-section. Subsequently, the paper presents the element formulation in a local Eulerian system (Izzuddin \& Elnashai, 1993-b), where the cross-section response over the element length is transformed into local element forces and tangent stiffness. This 
transformation is established through utilising the constant axial force criterion, and assuming quartic shape functions for the transverse displacements. Furthermore, the use of the proposed elastic element as an essential component within adaptive elasto-plastic analysis of 3D R/C frames is discussed, where the considerable modelling and computational benefits of the overall adaptive method are highlighted. Finally, several verification examples, using the nonlinear analysis program ADAPTIC (1991), are presented to demonstrate the accuracy and efficiency of the proposed beam-column formulation in the elastic analysis of 3D R/C frames.

\section{PURPOSE AND ASSUMPTIONS}

The proposed one-dimensional formulation is intended for modelling the elastic response of 3D R/C beam-columns using only one element per member, thus providing an essential ingredient for adaptive elasto-plastic analysis of 3D R/C frames (Izzuddin \& Lloyd Smith, 2000). Accordingly, this formulation does not in itself cater for such inelastic phenomena as the yielding of steel, compressive softening of concrete and the general hysteretic response of the two materials, all of which are instead considered on the level of the overall adaptive elasto-plastic analysis method, as discussed in Section 5.

The following assumptions are made in the new formulation:

1. Plane sections remain plane and normal to the longitudinal axis after flexural deformation; i.e. shear deformation is ignored.

2. Full strain compatibility exists between the steel reinforcement and the surrounding concrete; i.e. bond-slip between concrete and reinforcement is not considered.

3. The effect of concrete cracking on the torsional stiffness is ignored. Warping strains due to non-uniform torsion are neglected.

4. Concrete is homogeneous in nature. Its uniaxial compressive response is parabolic in the elastic range. It cannot sustain tensile stresses, which is consistent with 
conventional R/C section analysis (Morgan, 1971), where only the steel reinforcement provides tensile resistance. This simplified treatment of the tensile concrete response is justified by the fact that the proposed formulation, while elastic, is intended for use in adaptive elasto-plastic analysis of 3D R/C frames, principally in connection with the assessment of the ultimate limit state.

5. Steel reinforcement can vary over the element length. The response of steel in the elastic range is linear, and the reinforcement does not buckle under compression.

\section{CROSS-SECTION RESPONSE}

With the previous assumptions, the response characteristics of a $\mathrm{R} / \mathrm{C}$ cross-section can be fully defined in terms of four generalised strains (two biaxial curvatures, axial strain and twist) and four corresponding generalised stresses (two biaxial moments, axial force and torque). The relationship between the cross-sectional twist and torque is linear utilising the St. Venant's torsion constant. The relationship between the remaining three generalised stresses and strains depend on the chosen uniaxial material models for concrete and steel and the geometric representation of the cross-section, as discussed hereafter.

\subsection{Material Models}

For the steel reinforcement, a linear uniaxial stress-strain relationship is adopted in the elastic range:

$$
\sigma_{s}=\mathrm{E}_{s} \varepsilon_{s}
$$

where, $\mathrm{E}_{\mathrm{s}}=$ Young's modulus of steel.

For concrete, tensile stresses are ignored, and a piecewise parabolic-constant stress-strain relationship is assumed in the compressive range, as illustrated in Fig. 1. Although it is well established that the resistance of concrete reduces considerably beyond the crushing strain 
$\left(\varepsilon_{\mathrm{co}}\right)$, the proposed elastic formulation is not applicable for this range of strains, since the response of concrete becomes elasto-plastic for strains approaching $\varepsilon_{\mathrm{co}}$. Consequently, a constant resistance is assumed beyond $\varepsilon_{\mathrm{co}}$ merely to provide numerical stability for the proposed formulation. In an elasto-plastic analysis utilising the proposed elastic formulation, the adaptive analysis technique would automatically replace an elastic element with fibre type elasto-plastic elements (Izzuddin \& Elnashai, 1993-a) when it becomes inapplicable during analysis (Karayannis et al., 1994).

The stress-strain relationship for concrete is conveniently expressed as the sum of two piecewise zero-parabolic functions:

$$
\sigma_{\mathrm{c}}=\sigma_{c 1}\left(\varepsilon_{\mathrm{c}}\right)+\sigma_{c 2}\left(\varepsilon_{\mathrm{c}}+\varepsilon_{\mathrm{co}}\right)
$$

where,

$$
\left.\begin{array}{ll}
\sigma_{c l}\left(\varepsilon_{\mathrm{c}}\right)=\mathrm{kf}_{\mathrm{c}}\left[2 \frac{\varepsilon_{\mathrm{c}}}{\varepsilon_{\mathrm{co}}}+\left(\frac{\varepsilon_{\mathrm{c}}}{\varepsilon_{\mathrm{co}}}\right)^{2}\right] & \text { for } \varepsilon_{\mathrm{c}} \leq 0.0 \\
\sigma_{c l}\left(\varepsilon_{\mathrm{c}}\right)=0.0 & \text { for } \varepsilon_{\mathrm{c}}>0.0
\end{array}\right\}
$$

and,

$$
\left.\begin{array}{ll}
\sigma_{c 2}\left(\varepsilon_{\mathrm{p}}\right)=-\mathrm{kf}_{\mathrm{c}}\left(\frac{\varepsilon_{\mathrm{p}}}{\varepsilon_{\mathrm{co}}}\right)^{2} & \text { for } \varepsilon_{\mathrm{p}} \leq 0.0 \\
\sigma_{c 2}\left(\varepsilon_{\mathrm{p}}\right)=0.0 & \text { for } \varepsilon_{\mathrm{p}}>0.0
\end{array}\right\}
$$

in which, $\mathrm{k}=$ confinement factor, $\mathrm{f}_{\mathrm{c}}=$ concrete compressive strength, and $\varepsilon_{\mathrm{co}}=$ crushing strain of concrete.

\subsection{Geometric Representation}

In the proposed $\mathrm{R} / \mathrm{C}$ formulation, the overall cross-section response is obtained from evaluating the contributions of the concrete material and the steel reinforcement. To establish the contribution of the concrete for a general R/C cross-section, the cross-section is divided 
into a minimum number of rectangular areas over each of which the confinement factor can be assumed to be constant. This process is illustrated in Fig. 2 for a R/C T-section with different confinement factors $k_{i}$ for the inner core and $k_{0}$ for the outer cover. The overall cross-section response can be represented as the sum of the individual contributions of four rectangular concrete blocks, the first two having a confinement factor $\mathrm{k}_{\mathrm{o}}$ and the last two having a confinement factor $\left(\mathrm{k}_{\mathrm{i}}-\mathrm{k}_{\mathrm{o}}\right)$, and the contribution of the steel reinforcement. The problem of determining the overall response of a $\mathrm{R} / \mathrm{C}$ cross-section is reduced to establishing the response of a rectangular concrete block with a specific eccentricity from the origin of reference as well as the response of steel reinforcement, as discussed in detail hereafter.

\subsection{Generalised Response}

Taking the geometric centroid of a R/C cross-section as the origin of reference, the strain state of the cross-section, associated with normal uniaxial strains, can be fully defined by three independent generalised strains: the centroidal axial strain $\varepsilon_{\mathrm{a}}$ and the biaxial curvatures $\kappa_{\mathrm{y}}$ and $\kappa_{\mathrm{z}}$. The constant axial force criterion is used to determine $\varepsilon_{\mathrm{a}}$, as discussed in a later section on the element level, whereas $\kappa_{\mathrm{y}}$ and $\kappa_{\mathrm{z}}$ are simply the second derivatives of the transverse displacements $\mathrm{v}(\mathrm{x})$ and $\mathrm{w}(\mathrm{x})$ of the element longitudinal reference line:

$$
\begin{aligned}
& \kappa_{\mathrm{y}}=\mathrm{v}^{\prime \prime}(\mathrm{x}) \\
& \kappa_{\mathrm{z}}=\mathrm{w}^{\prime \prime}(\mathrm{x})
\end{aligned}
$$

The three corresponding generalised stresses for the overall cross-section are determined from the contributions of the steel reinforcement and the rectangular concrete blocks:

$$
\begin{aligned}
& \mathrm{f}_{\mathrm{a}}=\mathrm{f}_{\mathrm{as}}+\sum_{\mathrm{i}=1}^{\mathrm{n}_{\mathrm{c}}} \mathbf{f}_{\mathrm{ac}, \mathrm{i}} \\
& \mathrm{m}_{\mathrm{y}}=\mathrm{m}_{\mathrm{ys}}+\sum_{\mathrm{i}=1}^{\mathrm{n}_{\mathrm{c}}} \mathbf{m}_{\mathrm{yc}, \mathrm{i}} \\
& \mathrm{m}_{\mathrm{z}}=\mathrm{m}_{\mathrm{zs}}+\sum_{\mathrm{i}=1}^{\mathrm{n}_{\mathrm{c}}} \mathbf{m}_{\mathrm{zc}, \mathrm{i}}
\end{aligned}
$$


where,

$\mathrm{f}_{\mathrm{a}}, \mathrm{m}_{\mathrm{y}}, \mathrm{m}_{\mathrm{z}}=$ axial force and biaxial moments about centroid,

$\mathrm{f}_{\mathrm{as}}, \mathrm{m}_{\mathrm{ys}}, \mathrm{m}_{\mathrm{zs}}=$ axial force and biaxial bending moments of the steel reinforcement,

$\mathbf{f}_{\mathrm{ac}, \mathrm{i}}, \mathbf{m}_{\mathrm{yc}, \mathrm{i}}, \mathbf{m}_{\mathrm{zc}, \mathrm{i}}=$ axial force and biaxial bending moments of concrete block (i), and

$\mathrm{n}_{\mathrm{c}}=$ number of rectangular concrete blocks.

$\left(\mathrm{f}_{\mathrm{as}}, \mathrm{m}_{\mathrm{ys}}, \mathrm{m}_{\mathrm{zs}}\right)$ and $\left(\mathbf{f}_{\mathrm{ac}, \mathrm{i}}, \mathbf{m}_{\mathrm{yc}, \mathrm{i}}, \mathbf{m}_{\mathrm{zc}, \mathrm{i}}\right)$ are determined from the generalised strains $\left(\varepsilon_{\mathrm{a}}, \kappa_{\mathrm{y}}, \kappa_{\mathrm{z}}\right)$, as discussed in the next two sections.

The generalised tangent stiffness matrix ${ }_{\mathrm{t}} \mathbf{k}$ reflects the infinitesimal variation of the generalised stresses with the generalised strains on the overall cross-section level:

$$
\mathbf{k}=\left[\begin{array}{lll}
\frac{\partial \mathrm{f}_{\mathrm{a}}}{\partial \varepsilon_{\mathrm{a}}} & \frac{\partial \mathrm{f}_{\mathrm{a}}}{\partial \kappa_{\mathrm{y}}} & \frac{\partial \mathrm{f}_{\mathrm{a}}}{\partial \kappa_{\mathrm{z}}} \\
\frac{\partial \mathrm{m}_{\mathrm{y}}}{\partial \varepsilon_{\mathrm{a}}} & \frac{\partial \mathrm{m}_{\mathrm{y}}}{\partial \kappa_{\mathrm{y}}} & \frac{\partial \mathrm{m}_{\mathrm{y}}}{\partial \kappa_{\mathrm{z}}} \\
\frac{\partial \mathrm{m}_{\mathrm{z}}}{\partial \varepsilon_{\mathrm{a}}} & \frac{\partial \mathrm{m}_{\mathrm{z}}}{\partial \kappa_{\mathrm{y}}} & \frac{\partial \mathrm{m}_{\mathrm{z}}}{\partial \kappa_{\mathrm{z}}}
\end{array}\right]
$$

Again, ${ }_{t} \mathbf{k}$ is obtained from the contributions of the steel reinforcement and the concrete rectangular blocks:

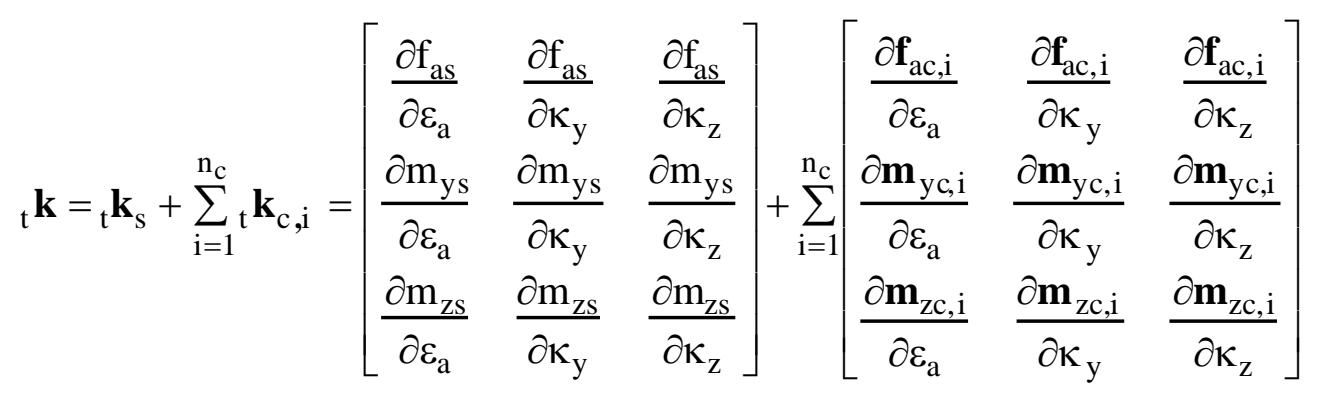

which are obtained explicitly in the following two sections.

\subsection{Response of Steel Reinforcement}


Given a set of generalised strains $\left(\varepsilon_{\mathrm{a}}, \kappa_{\mathrm{y}}, \kappa_{\mathrm{z}}\right)$, the contribution of steel reinforcement to the generalised stresses is obtained from:

$$
\begin{aligned}
& \boldsymbol{\varepsilon}_{\mathrm{s}, \mathrm{i}}=\boldsymbol{\varepsilon}_{\mathrm{a}}-\kappa_{\mathrm{y}} \mathbf{y}_{\mathrm{s}, \mathrm{i}}-\kappa_{\mathrm{z}} \mathbf{z}_{\mathrm{s}, \mathrm{i}} \quad \mathrm{i}=1, \mathrm{n}_{\mathrm{s}} \\
& \mathrm{f}_{\mathrm{as}}=\mathrm{E}_{\mathrm{s}} \sum_{\mathrm{i}=1}^{\mathrm{n}_{\mathrm{s}}} \mathbf{A}_{\mathrm{s}, \mathrm{i}} \boldsymbol{\varepsilon}_{\mathrm{s}, \mathrm{i}} \\
& \mathrm{m}_{\mathrm{ys}}=-\mathrm{E}_{\mathrm{s}} \sum_{\mathrm{i}=1}^{\mathrm{n}_{\mathrm{s}}} \mathbf{A}_{\mathrm{s}, \mathrm{i}} \boldsymbol{\varepsilon}_{\mathrm{s}, \mathrm{i}} \mathbf{y}_{\mathrm{s}, \mathrm{i}} \\
& \mathrm{m}_{\mathrm{zs}}=-\mathrm{E}_{\mathrm{s}} \sum_{\mathrm{i}=1}^{\mathrm{n}_{\mathrm{s}}} \mathbf{A}_{\mathrm{s}, \mathrm{i}} \boldsymbol{\varepsilon}_{\mathrm{s}, \mathrm{i}} \mathbf{z}_{\mathrm{s}, \mathrm{i}}
\end{aligned}
$$

where, $\mathbf{A}_{\mathrm{s}, \mathrm{i}}, \mathbf{y}_{\mathrm{s}, \mathrm{i}}$ and $\mathbf{z}_{\mathrm{s}, \mathrm{i}}$ are the area and distances from the cross-section origin of steel bar (i), respectively, and $n_{s}$ is the number of reinforcement bars in the R/C cross-section.

The contribution of steel reinforcement to the generalised tangent stiffness can be established from (6), and is expressed as:

$$
{ }_{\mathrm{t}} \mathbf{k}_{\mathrm{s}}=\mathrm{E}_{\mathrm{s}} \sum_{\mathrm{i}=1}^{\mathrm{n}_{\mathrm{s}}} \mathbf{A}_{\mathrm{s}, \mathrm{i}}\left[\begin{array}{ccc}
1 & -\mathbf{y}_{\mathrm{s}, \mathrm{i}} & -\mathbf{z}_{\mathrm{s}, \mathrm{i}} \\
-\mathbf{y}_{\mathrm{s}, \mathrm{i}} & \mathbf{y}_{\mathrm{s}, \mathrm{i}}^{2} & \mathbf{y}_{\mathrm{s}, \mathrm{i}} \mathbf{z}_{\mathrm{s}, \mathrm{i}} \\
-\mathbf{z}_{\mathrm{s}, \mathrm{i}} & \mathbf{y}_{\mathrm{s}, \mathrm{i}} \mathbf{z}_{\mathrm{s}, \mathrm{i}} & \mathbf{z}_{\mathrm{s}, \mathrm{i}}^{2}
\end{array}\right]
$$

\subsection{Response of Concrete Block}

Consider a typical rectangular concrete block, as shown in Fig. 3. Considerable simplification in the explicit expressions for generalised stresses can be achieved through determining first the strains at the four block corners from the generalised strains:

$$
\boldsymbol{\varepsilon}_{\mathrm{c}, \mathrm{i}}=\varepsilon_{\mathrm{a}}-\kappa_{\mathrm{y}}\left(\mathrm{y}_{\mathrm{c}}^{\mathrm{o}}+\xi_{\mathrm{i}} \frac{\mathrm{a}}{2}\right)-\kappa_{\mathrm{z}}\left(\mathrm{z}_{\mathrm{c}}^{\mathrm{o}}+\eta_{\mathrm{i}} \frac{\mathrm{b}}{2}\right) \quad \mathrm{i}=1,4
$$

where, 


$$
\left\{\begin{array}{c}
\xi_{1} \\
\eta_{1}
\end{array}\right\}=\left\{\begin{array}{c}
-1 \\
-1
\end{array}\right\} ; \quad\left\{\begin{array}{l}
\xi_{2} \\
\eta_{2}
\end{array}\right\}=\left\{\begin{array}{c}
+1 \\
-1
\end{array}\right\} ; \quad\left\{\begin{array}{l}
\xi_{3} \\
\eta_{3}
\end{array}\right\}=\left\{\begin{array}{l}
+1 \\
+1
\end{array}\right\} ; \quad\left\{\begin{array}{l}
\xi_{4} \\
\eta_{4}
\end{array}\right\}=\left\{\begin{array}{l}
-1 \\
+1
\end{array}\right\}
$$

The contribution of the block to the generalised cross-section stresses can be conveniently determined with reference to the block maximal corner $(\mathrm{m})$, defined as the corner having the largest compressive strain. This process involves weighted integrals of $\sigma_{c}$ in (2.a) over the block area, each of which can be conveniently expressed as the sum of two weighted integrals of the piecewise zero-parabolic functions $\sigma_{c 1}$ and $\sigma_{c 2}$, as detailed hereafter. The transformation of the local block response about $(\mathrm{m})$ to generalised stresses and stiffness about the cross-section origin $(\mathrm{O})$ is considered in a subsequent section.

\subsubsection{Contribution of $\sigma_{c 1}$}

In order to achieve simplified expressions for the generalised stresses, three convenient parameters $\left(\varepsilon_{\mathrm{c}, \mathrm{m}}, \mathrm{r}_{\mathrm{c}}, \mathrm{s}_{\mathrm{c}}\right)$ are first introduced in terms of the block corner strains:

$$
\begin{aligned}
& \boldsymbol{\varepsilon}_{\mathrm{c}, \mathrm{m}}=\min \left(\boldsymbol{\varepsilon}_{\mathrm{c}, \mathrm{i}} \quad \mathrm{i}=1,4\right) \\
& \mathrm{r}_{\mathrm{c}}=\frac{\boldsymbol{\varepsilon}_{\mathrm{c}, \mathrm{n}}}{\boldsymbol{\varepsilon}_{\mathrm{c}, \mathrm{m}}} \\
& \mathrm{s}_{\mathrm{c}}=\frac{\boldsymbol{\varepsilon}_{\mathrm{c}, \mathrm{q}}}{\boldsymbol{\varepsilon}_{\mathrm{c}, \mathrm{m}}}
\end{aligned}
$$

where, $\boldsymbol{\varepsilon}_{\mathrm{c}, \mathrm{m}}$ is the maximum corner compressive strain (minimum algebraic value), the corresponding corner identified as the maximal corner $(\mathrm{m})$. The remaining two terms $r_{c}$ and $\mathrm{s}_{\mathrm{c}}$ are ratios relative to $\boldsymbol{\varepsilon}_{\mathrm{c}, \mathrm{m}}$ of strains at the two corners $\mathrm{n}$ and $\mathrm{q}$ adjacent to $\mathrm{m}$, in the $\mathrm{y}$ and $\mathrm{z}$ directions, respectively.

The above three parameters can be used to define 6 different cases of integration domain over the rectangular block where the function $\sigma_{c l}$ is non-zero, as illustrated in Fig. 4 for a maximal bottom left corner: 
Case $1: \boldsymbol{\varepsilon}_{\mathrm{c}, \mathrm{m}} \geq 0$

Case 2: $\boldsymbol{\varepsilon}_{\mathrm{c}, \mathrm{m}}<0, \quad \mathrm{r}_{\mathrm{c}} \leq 0, \quad \mathrm{~s}_{\mathrm{c}} \leq 0$

Case $3: \boldsymbol{\varepsilon}_{\mathrm{c}, \mathrm{m}}<0, \quad \mathrm{r}_{\mathrm{c}}>0, \quad \mathrm{~s}_{\mathrm{c}} \leq 0$

Case $4: \boldsymbol{\varepsilon}_{\mathrm{c}, \mathrm{m}}<0, \quad \mathrm{r}_{\mathrm{c}} \leq 0, \quad \mathrm{~s}_{\mathrm{c}}>0$

Case 5: $\boldsymbol{\varepsilon}_{\mathrm{c}, \mathrm{m}}<0, \quad \mathrm{r}_{\mathrm{c}}>0, \quad \mathrm{~s}_{\mathrm{c}}>0, \quad \mathrm{r}_{\mathrm{c}}+\mathrm{s}_{\mathrm{c}}-1<0$

Case $6: \boldsymbol{\varepsilon}_{\mathrm{c}, \mathrm{m}}<0, \quad \mathrm{r}_{\mathrm{c}}>0, \quad \mathrm{~s}_{\mathrm{c}}>0, \quad \mathrm{r}_{\mathrm{c}}+\mathrm{s}_{\mathrm{c}}-1 \geq 0$
(Nil domain)

(Triangular domain)

(Trapezoidal domain)

(Trapezoidal domain)

The intersection of the domain boundary with the block edges meeting at the maximal corner is identified by two dimensions, obtained as:

$$
\begin{aligned}
& \mathrm{a}_{\mathrm{c}}=\frac{\mathrm{a}}{1-\mathrm{r}_{\mathrm{c}}} \\
& \mathrm{b}_{\mathrm{c}}=\frac{\mathrm{b}}{1-\mathrm{s}_{\mathrm{c}}}
\end{aligned}
$$

Case 1 is trivial corresponding to a zero contribution of function $\sigma_{c l}$ to the generalised stresses and stiffness.

Case 2 involves integration over a triangular domain, and leads to the following contribution of $\sigma_{c l}$ to generalised stresses about the block maximal corner (m):

$$
\begin{aligned}
& \mathrm{f}_{\mathrm{ac} 1}^{\mathrm{m}}=\frac{\mathrm{kf}_{\mathrm{c}} \boldsymbol{\varepsilon}_{\mathrm{c}, \mathrm{m}}\left(4 \varepsilon_{\mathrm{co}}+\boldsymbol{\varepsilon}_{\mathrm{c}, \mathrm{m}}\right) \mathrm{a}_{\mathrm{c}} \mathrm{b}_{\mathrm{c}}}{12 \varepsilon_{\mathrm{co}}^{2}} \\
& \mathrm{~m}_{\mathrm{yc} 1}^{\mathrm{m}}=\frac{-\mathrm{kf}_{\mathrm{c}} \boldsymbol{\varepsilon}_{\mathrm{c}, \mathrm{m}}\left(5 \varepsilon_{\mathrm{co}}+\boldsymbol{\varepsilon}_{\mathrm{c}, \mathrm{m}}\right) \mathrm{a}_{\mathrm{c}}^{2} \mathrm{~b}_{\mathrm{c}}}{60 \varepsilon_{\mathrm{co}}^{2}} \\
& \mathrm{~m}_{\mathrm{zc} 1}^{\mathrm{m}}=\frac{-\mathrm{kf}_{\mathrm{c}} \boldsymbol{\varepsilon}_{\mathrm{c}, \mathrm{m}}\left(5 \varepsilon_{\mathrm{co}}+\boldsymbol{\varepsilon}_{\mathrm{c}, \mathrm{m}}\right) \mathrm{a}_{\mathrm{c}} \mathrm{b}_{\mathrm{c}}^{2}}{60 \varepsilon_{\mathrm{co}}^{2}}
\end{aligned}
$$

The local block generalised tangent stiffness reflects the change in the generalised stresses with respect to generalised strains about $(\mathrm{m})$. Noting the following relationship between the three parameters $\left(\varepsilon_{\mathrm{c}, \mathrm{m}}, \mathrm{r}_{\mathrm{c}}, \mathrm{s}_{\mathrm{c}}\right)$ and the three generalised strains about $(\mathrm{m})$ :

$$
\begin{aligned}
& \boldsymbol{\varepsilon}_{\mathrm{c}, \mathrm{m}}=\varepsilon_{\mathrm{a}}^{\mathrm{m}} \\
& \mathrm{r}_{\mathrm{c}}=\frac{\varepsilon_{\mathrm{a}}^{\mathrm{m}}-\kappa_{\mathrm{y}}^{\mathrm{m}} \mathrm{a}}{\varepsilon_{\mathrm{a}}^{\mathrm{m}}}
\end{aligned}
$$




$$
\mathrm{s}_{\mathrm{c}}=\frac{\varepsilon_{\mathrm{a}}^{\mathrm{m}}-\kappa_{\mathrm{z}}^{\mathrm{m}} \mathrm{b}}{\varepsilon_{\mathrm{a}}^{\mathrm{m}}}
$$

and considering (9), (11) and (12), the generalised stiffness and can be established for Case 2 as:

$$
\begin{aligned}
& \frac{\partial \mathrm{f}_{\mathrm{ac} 1}^{\mathrm{m}}}{\partial \varepsilon_{\mathrm{a}}^{\mathrm{m}}}=\frac{\mathrm{kf}_{\mathrm{c}}\left(3 \varepsilon_{\mathrm{co}}+\varepsilon_{\mathrm{c}, \mathrm{m}}\right) \mathrm{a}_{\mathrm{c}} \mathrm{b}_{\mathrm{c}}}{3 \varepsilon_{\mathrm{co}}^{2}} \\
& \frac{\partial \mathrm{f}_{\mathrm{ac} 1}^{\mathrm{m}}}{\partial \kappa_{\mathrm{y}}^{\mathrm{m}}}=\frac{\partial \mathrm{m}_{\mathrm{yc} 1}^{\mathrm{m}}}{\partial \varepsilon_{\mathrm{a}}^{\mathrm{m}}}=\frac{-\mathrm{kf}_{\mathrm{c}}\left(4 \varepsilon_{\mathrm{co}}+\varepsilon_{\mathrm{c}, \mathrm{m}}\right) \mathrm{a}_{\mathrm{c}}^{2} \mathrm{~b}_{\mathrm{c}}}{12 \varepsilon_{\mathrm{co}}^{2}} \\
& \frac{\partial \mathrm{f}_{\mathrm{ac} 1}^{\mathrm{m}}}{\partial \kappa_{\mathrm{z}}^{\mathrm{m}}}=\frac{\partial \mathrm{m}_{\mathrm{zc} 1}^{\mathrm{m}}}{\partial \varepsilon_{\mathrm{a}}^{\mathrm{m}}}=\frac{-\mathrm{kf} \mathrm{f}_{\mathrm{c}}\left(4 \varepsilon_{\mathrm{co}}+\varepsilon_{\mathrm{c}, \mathrm{m}}\right) \mathrm{a}_{\mathrm{c}} \mathrm{b}_{\mathrm{c}}^{2}}{12 \varepsilon_{\mathrm{co}}^{2}} \\
& \frac{\partial \mathrm{m}_{\mathrm{yc} 1}^{\mathrm{m}}}{\partial \kappa_{\mathrm{y}}^{\mathrm{m}}}=\frac{\mathrm{kf} \mathrm{f}_{\mathrm{c}}\left(5 \varepsilon_{\mathrm{co}}+\varepsilon_{\mathrm{c}, \mathrm{m}}\right) \mathrm{a}_{\mathrm{c}}^{3} \mathrm{~b}_{\mathrm{c}}}{30 \varepsilon_{\mathrm{co}}^{2}} \\
& \frac{\partial \mathrm{m}_{\mathrm{yc} 1}^{\mathrm{m}}}{\partial \kappa_{\mathrm{z}}^{\mathrm{m}}}=\frac{\partial \mathrm{m}_{\mathrm{zc} 1}^{\mathrm{m}}}{\partial \kappa_{\mathrm{y}}^{\mathrm{m}}}=\frac{\mathrm{kf}_{\mathrm{c}}\left(5 \varepsilon_{\mathrm{co}}+\varepsilon_{\mathrm{c}, \mathrm{m}}\right) \mathrm{a}_{\mathrm{c}}^{2} \mathrm{~b}_{\mathrm{c}}^{2}}{60 \varepsilon_{\mathrm{co}}^{2}} \\
& \frac{\partial \mathrm{m}_{\mathrm{zc} 1}^{\mathrm{m}}}{\partial \kappa_{\mathrm{z}}^{\mathrm{m}}}=\frac{\mathrm{kf}_{\mathrm{c}}\left(5 \varepsilon_{\mathrm{co}}+\varepsilon_{\mathrm{c}, \mathrm{m}}\right) \mathrm{a}_{\mathrm{c}} \mathrm{b}_{\mathrm{c}}^{3}}{30 \varepsilon_{\mathrm{co}}^{2}}
\end{aligned}
$$

The generalised stresses for the remaining 4 cases of integration domain can be established through various combination of integrals over triangular domains. For example, cases (3) and (4) involve the integration over a trapezoidal domain, each of which can be expressed as the difference between two integrals over triangular domains, as illustrated for case (3) in Fig. 5. Similarly, the integral for case (5) can be expressed as the difference between an integral over a large triangular domain and two integrals over smaller triangular domains. Finally, the integral for case (6) can be obtained from a combination of integrals over four triangular domains. With the generalised stresses about (m) established explicitly, the generalised stiffness can be derived employing (9), (11), (12) and (13), as for case (2). 


\subsubsection{Contribution of $\sigma_{c 2}$}

Since the function $\sigma_{c 2}$ in (2.a) is non-zero only for a compressive concrete strain exceeding $\varepsilon_{\text {co }}$, the three parameters of the previous section are modified to account for this consideration:

$$
\begin{aligned}
& \boldsymbol{\varepsilon}_{\mathrm{p}, \mathrm{m}}=\min \left(\boldsymbol{\varepsilon}_{\mathrm{p}, \mathrm{i}} \quad \mathrm{i}=1,4\right) \\
& \mathrm{r}_{\mathrm{p}}=\frac{\boldsymbol{\varepsilon}_{\mathrm{p}, \mathrm{n}}}{\boldsymbol{\varepsilon}_{\mathrm{p}, \mathrm{m}}} \\
& \mathrm{s}_{\mathrm{p}}=\frac{\boldsymbol{\varepsilon}_{\mathrm{p}, \mathrm{q}}}{\boldsymbol{\varepsilon}_{\mathrm{p}, \mathrm{n}}}
\end{aligned}
$$

where,

$$
\boldsymbol{\varepsilon}_{\mathrm{p}, \mathrm{i}}=\boldsymbol{\varepsilon}_{\mathrm{c}, \mathrm{i}}+\varepsilon_{\mathrm{co}} \quad \mathrm{i}=1,4
$$

Again, 6 cases of integration domain can be identified according to conditions identical to (10), but using the modified parameters $\left(\varepsilon_{\mathrm{p}, \mathrm{m}}, \mathrm{r}_{\mathrm{p}}, \mathrm{s}_{\mathrm{p}}\right)$. Similarly, expressions for $\mathrm{a}_{\mathrm{p}}$ and $\mathrm{b}_{\mathrm{p}}$ identical to (11), but in terms of parameters $r_{p}$ and $s_{p}$, can be used to establish the intersection of the domain boundary with the edges meeting at the maximal corner.

As previously for function $\sigma_{c l}$, case 1 corresponds to a zero contribution for function $\sigma_{c 2}$ to the generalised stresses and stiffness about (m).

For case 2, the contribution to the generalised stresses about (m) can be obtained as:

$$
\begin{aligned}
& \mathrm{f}_{\mathrm{ac} 2}^{\mathrm{m}}=\frac{-\mathrm{kf}_{\mathrm{c}} \varepsilon_{\mathrm{p}, \mathrm{m}}^{2} \mathrm{a}_{\mathrm{p}} \mathrm{b}_{\mathrm{p}}}{12 \varepsilon_{\mathrm{co}}^{2}} \\
& \mathrm{~m}_{\mathrm{yc} 2}^{\mathrm{m}}=\frac{\mathrm{kf}_{\mathrm{c}} \varepsilon_{\mathrm{p}, \mathrm{m}}^{2} \mathrm{a}_{\mathrm{p}}^{2} \mathrm{~b}_{\mathrm{p}}}{60 \varepsilon_{\mathrm{co}}^{2}} \\
& \mathrm{~m}_{\mathrm{zc} 2}^{\mathrm{m}}=\frac{\mathrm{kf}_{\mathrm{c}} \varepsilon_{\mathrm{p}, \mathrm{m}}^{2} \mathrm{a}_{\mathrm{p}} \mathrm{b}_{\mathrm{p}}^{2}}{60 \varepsilon_{\mathrm{co}}^{2}}
\end{aligned}
$$


with the generalised stiffness about $(\mathrm{m})$ given by:

$$
\begin{aligned}
& \frac{\partial \mathrm{f}_{\mathrm{ac} 2}^{\mathrm{m}}}{\partial \varepsilon_{\mathrm{a}}^{\mathrm{m}}}=\frac{-\mathrm{kf}_{\mathrm{c}} \boldsymbol{\varepsilon}_{\mathrm{p}, \mathrm{m}} \mathrm{a}_{\mathrm{p}} \mathrm{b}_{\mathrm{p}}}{3 \varepsilon_{\mathrm{co}}^{2}} \\
& \frac{\partial \mathrm{f}_{\mathrm{ac} 2}^{\mathrm{m}}}{\partial \mathrm{\kappa}_{\mathrm{y}}^{\mathrm{m}}}=\frac{\partial \mathrm{m}_{\mathrm{yc} 2}^{\mathrm{m}}}{\partial \varepsilon_{\mathrm{a}}^{\mathrm{m}}}=\frac{\mathrm{kf} \mathrm{f}_{\mathrm{c}, \mathrm{m}} \mathrm{a}_{\mathrm{p}}^{2} \mathrm{~b}_{\mathrm{p}}}{12 \varepsilon_{\mathrm{co}}^{2}} \\
& \frac{\partial \mathrm{f}_{\mathrm{ac} 2}^{\mathrm{m}}}{\partial \kappa_{\mathrm{z}}^{\mathrm{m}}}=\frac{\partial \mathrm{m}_{\mathrm{zc} 2}^{\mathrm{m}}}{\partial \varepsilon_{\mathrm{a}}^{\mathrm{m}}}=\frac{\mathrm{kf} \mathrm{f}_{\mathrm{c}} \boldsymbol{\varepsilon}_{\mathrm{p}, \mathrm{m}} \mathrm{a}_{\mathrm{p}} \mathrm{b}_{\mathrm{p}}^{2}}{12 \varepsilon_{\mathrm{co}}^{2}} \\
& \frac{\partial \mathrm{m}_{\mathrm{yc} 2}^{\mathrm{m}}}{\partial \kappa_{\mathrm{y}}^{\mathrm{m}}}=\frac{-\mathrm{kf} \mathrm{f}_{\mathrm{c}} \boldsymbol{\varepsilon}_{\mathrm{p}, \mathrm{m}} \mathrm{a}_{\mathrm{p}}^{3} \mathrm{~b}_{\mathrm{p}}}{30 \varepsilon_{\mathrm{co}}^{2}} \\
& \frac{\partial \mathrm{m}_{\mathrm{yc} 2}^{\mathrm{m}}}{\partial \kappa_{\mathrm{z}}^{\mathrm{m}}}=\frac{\partial \mathrm{m}_{\mathrm{zc} 2}^{\mathrm{m}}}{\partial \kappa_{\mathrm{y}}^{\mathrm{m}}}=\frac{-\mathrm{kf} \mathrm{f}_{\mathrm{c}} \boldsymbol{\varepsilon}_{\mathrm{p}, \mathrm{m}} \mathrm{a}_{\mathrm{p}}^{2} \mathrm{~b}_{\mathrm{p}}^{2}}{60 \varepsilon_{\mathrm{co}}^{2}} \\
& \frac{\partial \mathrm{m}_{\mathrm{zc} 2}^{\mathrm{m}}}{\partial \kappa_{\mathrm{z}}^{\mathrm{m}}}=\frac{-\mathrm{kf}_{\mathrm{c}} \boldsymbol{\varepsilon}_{\mathrm{p}, \mathrm{m}} \mathrm{a}_{\mathrm{p}} \mathrm{b}_{\mathrm{p}}^{3}}{30 \varepsilon_{\mathrm{co}}^{2}}
\end{aligned}
$$

The contribution of function $\sigma_{c 2}$ for the remaining 4 cases of integration domain can be determined in a similar manner to that of function $\sigma_{c l}$ outlined in the previous section.

\subsubsection{Transformation to cross-section origin}

The contribution of a concrete block to generalised stresses and stiffness about the maximal corner (m) is the sum of the contributions from functions $\sigma_{c 1}$ and $\sigma_{c 2}$ established in the previous two sections:

$$
\begin{aligned}
& \mathrm{f}_{\mathrm{ac}}^{\mathrm{m}}=\mathrm{f}_{\mathrm{ac} 1}^{\mathrm{m}}+\mathrm{f}_{\mathrm{ac} 2}^{\mathrm{m}} \\
& \mathrm{m}_{\mathrm{yc}}^{\mathrm{m}}=\mathrm{m}_{\mathrm{yc} 1}^{\mathrm{m}}+\mathrm{m}_{\mathrm{yc} 2}^{\mathrm{m}} \\
& \mathrm{m}_{\mathrm{zc}}^{\mathrm{m}}=\mathrm{m}_{\mathrm{zc} 1}^{\mathrm{m}}+\mathrm{m}_{\mathrm{zc} 2}^{\mathrm{m}}
\end{aligned}
$$




$$
{ }_{\mathrm{t}} \mathbf{k}_{\mathrm{c}}^{\mathrm{m}}=\left[\begin{array}{ccc}
\frac{\partial \mathrm{f}_{\mathrm{ac} 1}^{\mathrm{m}}}{\partial \varepsilon_{\mathrm{a}}^{\mathrm{m}}} & \frac{\partial \mathrm{f}_{\mathrm{ac} 1}^{\mathrm{m}}}{\partial \kappa_{\mathrm{y}}^{\mathrm{m}}} & \frac{\partial \mathrm{f}_{\mathrm{ac} 1}^{\mathrm{m}}}{\partial \kappa_{\mathrm{z}}^{\mathrm{m}}} \\
\frac{\partial \mathrm{m}_{\mathrm{yc} 1}^{\mathrm{m}}}{\partial \varepsilon_{\mathrm{a}}^{\mathrm{m}}} & \frac{\partial \mathrm{m}_{\mathrm{yc} 1}^{\mathrm{m}}}{\partial \kappa_{\mathrm{y}}^{\mathrm{m}}} & \frac{\partial \mathrm{m}_{\mathrm{yc} 1}^{\mathrm{m}}}{\partial \kappa_{\mathrm{z}}^{\mathrm{m}}} \\
\frac{\partial \mathrm{m}_{\mathrm{zc} 1}^{\mathrm{m}}}{\partial \varepsilon_{\mathrm{a}}^{\mathrm{m}}} & \frac{\partial \mathrm{m}_{\mathrm{zc} 1}^{\mathrm{m}}}{\partial \kappa_{\mathrm{y}}^{\mathrm{m}}} & \frac{\partial \mathrm{m}_{\mathrm{zc} 1}^{\mathrm{m}}}{\partial \kappa_{\mathrm{z}}^{\mathrm{m}}}
\end{array}\right]+\left[\begin{array}{ccc}
\frac{\partial \mathrm{f}_{\mathrm{ac} 2}^{\mathrm{m}}}{\partial \varepsilon_{\mathrm{a}}^{\mathrm{m}}} & \frac{\partial \mathrm{f}_{\mathrm{ac} 2}^{\mathrm{m}}}{\partial \kappa_{\mathrm{y}}^{\mathrm{m}}} & \frac{\partial \mathrm{f}_{\mathrm{ac} 2}^{\mathrm{m}}}{\partial \kappa_{\mathrm{z}}^{\mathrm{m}}} \\
\frac{\partial \mathrm{m}_{\mathrm{yc} 2}^{\mathrm{m}}}{\partial \varepsilon_{\mathrm{a}}^{\mathrm{m}}} & \frac{\partial \mathrm{m}_{\mathrm{yc} 2}^{\mathrm{m}}}{\partial \kappa_{\mathrm{y}}^{\mathrm{m}}} & \frac{\partial \mathrm{m}_{\mathrm{yc} 2}^{\mathrm{m}}}{\partial \kappa_{\mathrm{z}}^{\mathrm{m}}} \\
\frac{\partial \mathrm{m}_{\mathrm{zc} 2}^{\mathrm{m}}}{\partial \varepsilon_{\mathrm{a}}^{\mathrm{m}}} & \frac{\partial \mathrm{m}_{\mathrm{zc} 2}^{\mathrm{m}}}{\partial \kappa_{\mathrm{y}}^{\mathrm{m}}} & \frac{\partial \mathrm{m}_{\mathrm{zc} 2}^{\mathrm{m}}}{\partial \kappa_{\mathrm{z}}^{\mathrm{m}}}
\end{array}\right]
$$

Noting the following relationship between the generalised strains about the cross-section origin $(\mathrm{O})$ and the maximal corner $(\mathrm{m})$ :

$$
\left\{\begin{array}{c}
\varepsilon_{\mathrm{a}}^{\mathrm{m}} \\
\kappa_{\mathrm{y}}^{\mathrm{m}} \\
\kappa_{\mathrm{z}}^{\mathrm{m}}
\end{array}\right\}=\mathbf{T}_{\mathrm{m}}\left\{\begin{array}{c}
\varepsilon_{\mathrm{a}} \\
\kappa_{\mathrm{y}} \\
\kappa_{\mathrm{z}}
\end{array}\right\}=\left[\begin{array}{ccc}
1 & -\mathrm{y}_{\mathrm{c}}^{\mathrm{o}}-\xi_{\mathrm{m}} \frac{\mathrm{a}}{2} & -\mathrm{z}_{\mathrm{c}}^{\mathrm{o}}-\eta_{\mathrm{m}} \frac{\mathrm{b}}{2} \\
0 & -\xi_{\mathrm{m}} & 0 \\
0 & 0 & -\eta_{\mathrm{m}}
\end{array}\right]\left\{\begin{array}{c}
\varepsilon_{\mathrm{a}} \\
\kappa_{\mathrm{y}} \\
\kappa_{\mathrm{z}}
\end{array}\right\}
$$

it can be shown that the contribution of a concrete block to generalised stresses and stiffness about $(\mathrm{O})$ can be determined from:

$$
\begin{aligned}
& \left\{\begin{array}{c}
\mathrm{f}_{\mathrm{ac}} \\
\mathrm{m}_{\mathrm{yc}} \\
\mathrm{m}_{\mathrm{zc}}
\end{array}\right\}=\mathbf{T}_{\mathrm{m}}^{\mathrm{T}}\left\{\begin{array}{c}
\mathrm{f}_{\mathrm{ac}}^{\mathrm{m}} \\
\mathrm{m}_{\mathrm{yc}}^{\mathrm{m}} \\
\mathrm{m}_{\mathrm{zc}}^{\mathrm{m}}
\end{array}\right\} \\
& { }_{\mathrm{t}} \mathbf{k}_{\mathrm{c}}=\mathbf{T}_{\mathrm{m}{ }_{\mathrm{t}} \mathbf{k}_{\mathrm{c}}^{\mathrm{m}} \mathbf{T}_{\mathrm{m}}}
\end{aligned}
$$

where, $\xi_{\mathrm{m}}$ and $\eta_{\mathrm{m}}$ appearing in the transformation matrix $\mathbf{T}_{\mathrm{m}}$ can be established from (8.b) for maximal corner $(\mathrm{m})$.

\section{FORMULATION ON THE ELEMENT LEVEL}

The proposed formulation is aimed at representing a whole elastic $\mathrm{R} / \mathrm{C}$ member with one element, which is partly accomplished through the adoption of higher-order quartic shape functions for the transverse displacements. In addition to redressing the inaccuracies of common cubic formulations, the proposed formulation departs from the conventional finiteelement approach in that the axial displacement is not represented by shape functions. Instead, 
the constant axial force criterion is adopted, which enforces axial equilibrium along the element length, the violation of which usually leads to an over-stiff element incapable of accurately representing a whole member.

\subsection{Kinematics}

The quartic formulation is derived in a local Eulerian system (Izzuddin \& Elnashai, 1993-b), and it utilises 8 local freedoms, as shown in Fig. 6. The element freedoms and corresponding forces are represented by the following vectors:

$$
\begin{aligned}
& { }_{c} \mathbf{u}=\left\langle\theta_{\mathrm{y} 1}, \theta_{\mathrm{y} 2}, \mathrm{t}_{\mathrm{y}}, \theta_{\mathrm{z} 1}, \theta_{\mathrm{z} 2}, \mathrm{t}_{\mathrm{z}}, \Delta, \theta_{\mathrm{T}}\right\rangle^{\mathrm{T}} \\
& { }_{\mathrm{c}} \mathbf{f}=\left\langle\mathrm{M}_{\mathrm{y} 1}, \mathrm{M}_{\mathrm{y} 2}, \mathrm{~T}_{\mathrm{y}}, \mathrm{M}_{\mathrm{z} 1}, \mathrm{M}_{\mathrm{z} 2}, \mathrm{~T}_{\mathrm{z}}, \mathrm{F}, \mathrm{M}_{\mathrm{T}}\right\rangle^{\mathrm{T}}
\end{aligned}
$$

Quartic shape functions are adopted for the two transverse displacements, given by:

$$
\begin{aligned}
\mathrm{v}(\mathrm{x})= & \mathrm{t}_{\mathrm{y}}-\left[\frac{\mathrm{L}}{4}\left(\theta_{\mathrm{y} 1}+\theta_{\mathrm{y} 2}\right)\right]\left(\frac{\mathrm{x}}{\mathrm{L}}\right)+\left[\frac{\mathrm{L}}{2}\left(\theta_{\mathrm{y} 1}-\theta_{\mathrm{y} 2}\right)-8 \mathrm{t}_{\mathrm{y}}\right]\left(\frac{\mathrm{x}}{\mathrm{L}}\right)^{2} \\
& \left.+\left[\mathrm{L}\left(\theta_{\mathrm{y} 1}+\theta_{\mathrm{y} 2}\right)\right]\left(\frac{\mathrm{x}}{\mathrm{L}}\right)^{3}+\left[2 \mathrm{~L}\left(\theta_{\mathrm{y} 2}-\theta_{\mathrm{y} 1}\right)+16 \mathrm{t}_{\mathrm{y}}\right] \frac{\mathrm{x}}{\mathrm{L}}\right)^{4} \\
\mathrm{w}(\mathrm{x})= & \mathrm{t}_{\mathrm{z}}-\left[\frac{\mathrm{L}}{4}\left(\theta_{\mathrm{z} 1}+\theta_{\mathrm{z} 2}\right)\right]\left(\frac{\mathrm{x}}{\mathrm{L}}\right)+\left[\frac{\mathrm{L}}{2}\left(\theta_{\mathrm{zl}}-\theta_{\mathrm{z} 2}\right)-8 \mathrm{t}_{\mathrm{z}}\right]\left(\frac{\mathrm{x}}{\mathrm{L}}\right)^{2} \\
& +\left[\mathrm{L}\left(\theta_{\mathrm{z} 1}+\theta_{\mathrm{z} 2}\right)\right]\left(\frac{\mathrm{x}}{\mathrm{L}}\right)^{3}+\left[2 \mathrm{~L}\left(\theta_{\mathrm{z} 2}-\theta_{\mathrm{z} 1}\right)+16 \mathrm{t}_{\mathrm{z}}\right]\left(\frac{\mathrm{x}}{\mathrm{L}}\right)^{4}
\end{aligned}
$$

where, $\mathrm{L}$ is the element length.

As noted previously, the generalised curvature strains $\kappa_{\mathrm{y}}$ and $\kappa_{\mathrm{z}}$ are the second derivatives of the transverse displacements:

$$
\kappa_{\mathrm{y}}=\mathrm{v}^{\prime \prime}(\mathrm{x})=\frac{1}{\mathrm{~L}}\left\{\theta_{\mathrm{y} 1}-\theta_{\mathrm{y} 2}-16\left(\frac{\mathrm{t}_{\mathrm{y}}}{\mathrm{L}}\right)+6\left(\theta_{\mathrm{y} 1}+\theta_{\mathrm{y} 2}\right)\left(\frac{\mathrm{x}}{\mathrm{L}}\right)+24\left[\theta_{\mathrm{y} 2}-\theta_{\mathrm{y} 1}+8\left(\frac{\mathrm{t}_{\mathrm{y}}}{\mathrm{L}}\right)\right]\left(\frac{\mathrm{x}}{\mathrm{L}}\right)^{2}\right\}
$$




$$
\kappa_{\mathrm{z}}=\mathrm{w}^{\prime \prime}(\mathrm{x})=\frac{1}{\mathrm{~L}}\left\{\theta_{\mathrm{z} 1}-\theta_{\mathrm{z} 2}-16\left(\frac{\mathrm{t}_{\mathrm{z}}}{\mathrm{L}}\right)+6\left(\theta_{\mathrm{z} 1}+\theta_{\mathrm{z} 2}\right)\left(\frac{\mathrm{x}}{\mathrm{L}}\right)+24\left[\theta_{\mathrm{z} 2}-\theta_{\mathrm{z} 1}+8\left(\frac{\mathrm{t}_{\mathrm{z}}}{\mathrm{L}}\right)\right]\left(\frac{\mathrm{x}}{\mathrm{L}}\right)^{2}\right\}
$$

The generalised axial strain $\varepsilon_{\mathrm{a}}$ includes the nonlinear effect of bending on axial stretching, as given by:

$$
\varepsilon_{\mathrm{a}}=\mathrm{u}^{\prime}+\frac{1}{2}\left(\mathrm{v}^{\prime 2}+\mathrm{w}^{\prime 2}\right)
$$

Since the axial displacement $\mathrm{u}(\mathrm{x})$ is not prescribed by means of shape functions, only an average generalised axial strain $\left(\varepsilon_{\mathrm{m}}\right)$ can be evaluated in terms of the element freedoms:

$$
\int_{-\mathrm{L} / 2}^{\mathrm{L} / 2} \varepsilon_{\mathrm{a}} \mathrm{dx}=\varepsilon_{\mathrm{m}} \mathrm{L}=\int_{-\mathrm{L} / 2}^{\mathrm{L} / 2} \mathrm{u}^{\prime} \mathrm{dx}+\frac{1}{2} \int_{-\mathrm{L} / 2}^{\mathrm{L} / 2}\left(\mathrm{v}^{\prime 2}+\mathrm{w}^{\prime 2}\right) \mathrm{dx}=\Delta+\frac{1}{2} \int_{-\mathrm{L} / 2}^{\mathrm{L} / 2}\left(\mathrm{v}^{\prime 2}+\mathrm{w}^{\prime 2}\right) \mathrm{dx}
$$

which in combination with (22) leads to:

$$
\begin{aligned}
\varepsilon_{\mathrm{m}}=\frac{\Delta}{\mathrm{L}}+\frac{1}{210}\{ & 8\left(\theta_{\mathrm{y} 1}^{2}+\theta_{\mathrm{y} 2}^{2}+\theta_{\mathrm{z} 1}^{2}+\theta_{\mathrm{z} 2}^{2}\right)+16\left(\theta_{\mathrm{y} 2}-\theta_{\mathrm{y} 1}\right)\left(\frac{\mathrm{t}_{\mathrm{y}}}{\mathrm{L}}\right) \\
+ & \left.16\left(\theta_{\mathrm{z} 2}-\theta_{\mathrm{z} 1}\right)\left(\frac{\mathrm{t}_{\mathrm{z}}}{\mathrm{L}}\right)+5\left(\theta_{\mathrm{y} 1} \theta_{\mathrm{y} 2}+\theta_{\mathrm{z} 1} \theta_{\mathrm{z} 2}\right)+512\left(\frac{\mathrm{t}_{\mathrm{y}}^{2}+\mathrm{t}_{\mathrm{z}}^{2}}{\mathrm{~L}^{2}}\right)\right\}
\end{aligned}
$$

The variation of $\varepsilon_{\mathrm{a}}$ along the element length is determined from the constant axial force criterion in combination with the average axial strain $\varepsilon_{\mathrm{m}}$, as discussed in the following section.

\subsection{Axial Equilibrium}

Since a general analytical solution for $\varepsilon_{\mathrm{a}}$ satisfying axial equilibrium is extremely difficult, the conditions of axial equilibrium will be considered for discrete positions over the element length. Conveniently, these are chosen to be the same as the Gauss stations used for numerical integration of the governing virtual work equations presented in the following section.

The procedure for determining the variation of $\varepsilon_{\mathrm{a}}$ is iterative, which is started by initialising $\boldsymbol{\varepsilon}_{\mathrm{a}, \mathrm{g}}$ for all Gauss points (g) to the corresponding values established at the end of the previous 
load step. Since the curvature generalised strains $\boldsymbol{\kappa}_{\mathrm{y}, \mathrm{g}}$ and $\boldsymbol{\kappa}_{\mathrm{z}, \mathrm{g}}$ are available from (23), the generalised axial forces $\mathbf{f}_{\mathrm{a}, \mathrm{g}}$ can be determined for all Gauss points according to Section 5. If these axial forces are not in equilibrium, $\boldsymbol{\varepsilon}_{\mathrm{a}, \mathrm{g}}$ is modified by iterative increments $\Delta \boldsymbol{\varepsilon}_{\mathrm{a}, \mathrm{g}}$ determined from the following equations:

$$
\begin{aligned}
& \mathbf{f}_{\mathrm{a}, \mathrm{g}}+{ }_{\mathrm{t}} \mathbf{k}_{1,1, \mathrm{~g}} \Delta \boldsymbol{\varepsilon}_{\mathrm{a}, \mathrm{g}}=\mathbf{f}_{\mathrm{a}, \mathrm{g}+1}+{ }_{\mathrm{t}} \mathbf{k}_{1,1, \mathrm{~g}+1} \Delta \boldsymbol{\varepsilon}_{\mathrm{a}, \mathrm{g}+1} \quad \mathrm{~g}=1, \mathrm{n}_{\mathrm{g}}-1 \\
& \sum_{\mathrm{g}=1}^{\mathrm{n}_{\mathrm{g}}} \mathbf{w}_{\mathrm{g}}\left(\boldsymbol{\varepsilon}_{\mathrm{a}, \mathrm{g}}+\Delta \boldsymbol{\varepsilon}_{\mathrm{a}, \mathrm{g}}\right)=\varepsilon_{\mathrm{m}} \mathrm{L}
\end{aligned}
$$

where, $\mathrm{n}_{\mathrm{g}}$ is the number of Gauss points and $\mathbf{w}_{\mathrm{g}}$ is the weighting factor for Gauss point $(\mathrm{g})$.

The first expression (26.a) provides $\left(\mathrm{n}_{\mathrm{g}}-1\right)$ equations of axial equilibrium between adjacent Gauss stations, whereas expression (26.b) provides a numerical integration equivalent to (25.a). With the availability of ${ }_{\mathrm{t}} \mathbf{k}_{1,1, \mathrm{~g}}$ according to Section 5 , these equations can be used to solve for the $\mathrm{n}_{\mathrm{g}}$ iterative increments $\Delta \boldsymbol{\varepsilon}_{\mathrm{a}, \mathrm{g}}$ :

$$
\begin{aligned}
& \Delta \boldsymbol{\varepsilon}_{\mathrm{a}, 1}=\left[\frac{\varepsilon_{\mathrm{m}} \mathrm{L}-\sum_{\mathrm{g}=1}^{\mathrm{n}_{\mathrm{g}}} \mathbf{w}_{\mathrm{g}}\left\{\boldsymbol{\varepsilon}_{\mathrm{a}, \mathrm{g}}+\frac{\mathbf{f}_{\mathrm{a}, 1}-\mathbf{f}_{\mathrm{a}, \mathrm{g}}}{{ }_{\mathrm{t}} \mathbf{k}_{1,1, \mathrm{~g}}}\right\}}{\sum_{\mathrm{g}=1}^{\mathrm{n}_{\mathrm{g}}} \mathbf{w}_{\mathrm{g}}\left\{\frac{{ }_{\mathrm{t}} \mathbf{k}_{1,1,1}}{{ }_{\mathrm{t}} \mathbf{k}_{1,1, \mathrm{~g}}}\right\}}\right] \\
& \Delta \boldsymbol{\varepsilon}_{\mathrm{a}, \mathrm{g}}=\frac{\left(\mathbf{f}_{\mathrm{a}, 1}-\mathbf{f}_{\mathrm{a}, \mathrm{g}}\right)+{ }_{\mathrm{t}} \mathbf{k}_{1,1,1} \Delta \boldsymbol{\varepsilon}_{\mathrm{a}, 1}}{{ }_{\mathrm{t}} \mathbf{k}_{1,1, \mathrm{~g}}}
\end{aligned}
$$

The iterative procedure is continued until the sum of the $\mathrm{n}_{\mathrm{g}}$ iterative increments $\Delta \boldsymbol{\varepsilon}_{\mathrm{a}, \mathrm{g}}$ in absolute value becomes very small relative to the average axial strain $\varepsilon_{\mathrm{m}}$. After convergence, axial equilibrium is considered to be satisfied, and the element axial force $F$ is set equal to $\mathbf{f}_{a, 1}$. The iterative strategy for enforcing the constant axial force criterion is illustrated in Fig. 7.

\subsection{Local Forces}

The proposed quartic formulation can be employed with an Eulerian large displacement approach (Izzuddin \& Elnashai, 1993-b), which requires the calculation of the basic local 
forces $\left\langle\mathrm{M}_{\mathrm{y} 1}, \mathrm{M}_{\mathrm{y} 2}, \mathrm{M}_{\mathrm{z} 1}, \mathrm{M}_{\mathrm{z} 2}, \mathrm{~F}, \mathrm{M}_{\mathrm{T}}\right\rangle$ corresponding to given values for the basic local freedoms $\left\{\theta_{\mathrm{y} 1}, \theta_{\mathrm{y} 2}, \theta_{\mathrm{z} 1}, \theta_{\mathrm{z} 2}, \Delta, \theta_{\mathrm{T}}\right\}$. Since the quartic formulation utilises two additional internal freedoms $\left\langle\mathrm{t}_{\mathrm{y}}, \mathrm{t}_{\mathrm{z}}\right\rangle$ to provide quartic shape functions for the transverse displacements, these internal freedoms are established using a process of static condensation. This involves an iterative procedure such that the corresponding resistance forces $\left\langle\mathrm{T}_{\mathrm{y}}, \mathrm{T}_{\mathrm{z}}\right\rangle$ are in equilibrium with equivalent loads $\left\langle\mathrm{T}_{\mathrm{yeq}}, \mathrm{T}_{\mathrm{zeq}}\right\rangle$ arising from internal element loading (Izzuddin, 1996). The iterative process is started with initialising $\left\langle\mathrm{t}_{\mathrm{y}}, \mathrm{t}_{\mathrm{z}}\right\rangle$ to values determined for the last load step, and then determining the iterative corrections $\left\langle\Delta \mathrm{t}_{\mathrm{y}}, \Delta \mathrm{t}_{\mathrm{z}}\right\rangle$ according to:

$$
\left\{\begin{array}{c}
\Delta t_{y} \\
\Delta t_{z}
\end{array}\right\}=\left[\begin{array}{cc}
{ }_{b} \mathbf{k}_{3,3} & { }_{b} \mathbf{k}_{3,6} \\
{ }_{b} \mathbf{k}_{6,3} & { }_{b} \mathbf{k}_{6,6}
\end{array}\right]^{-1}\left\{\begin{array}{c}
T_{\text {yeq }}-T_{y} \\
T_{\text {zeq }}-T_{z}
\end{array}\right\}
$$

where, ${ }_{\mathrm{b}} \mathbf{k}$ is the local tangent stiffness matrix discussed in the following section.

The resistance forces $\left\langle\mathrm{T}_{\mathrm{y}}, \mathrm{T}_{\mathrm{z}}\right\rangle$ in (28) are obtained from the virtual work equation, which is integrated numerically:

$$
\begin{aligned}
& \mathrm{T}_{\mathrm{y}}=\sum_{\mathrm{g}=1}^{\mathrm{n}_{\mathrm{g}}} \mathbf{w}_{\mathrm{g}}\left(\mathbf{m}_{\mathrm{y}, \mathrm{g}} \frac{\partial \mathbf{k}_{\mathrm{y}, \mathrm{g}}}{\partial \mathrm{t}_{\mathrm{y}}}+\mathbf{m}_{\mathrm{z}, \mathrm{g}} \frac{\partial \mathbf{k}_{\mathrm{z}, \mathrm{g}}}{\partial \mathrm{t}_{\mathrm{y}}}+\mathbf{f}_{\mathrm{a}, \mathrm{g}} \frac{\partial \boldsymbol{\varepsilon}_{\mathrm{a}, \mathrm{g}}}{\partial \mathrm{t}_{\mathrm{y}}}\right) \\
& \mathrm{T}_{\mathrm{z}}=\sum_{\mathrm{g}=1}^{\mathrm{n}_{\mathrm{g}}} \mathbf{w}_{\mathrm{g}}\left(\mathbf{m}_{\mathrm{y}, \mathrm{g}} \frac{\partial \boldsymbol{\kappa}_{\mathrm{y}, \mathrm{g}}}{\partial \mathrm{t}_{\mathrm{z}}}+\mathbf{m}_{\mathrm{z}, \mathrm{g}} \frac{\partial \mathbf{\kappa}_{\mathrm{z}, \mathrm{g}}}{\partial \mathrm{t}_{\mathrm{z}}}+\mathbf{f}_{\mathrm{a}, \mathrm{g}} \frac{\partial \boldsymbol{\varepsilon}_{\mathrm{a}, \mathrm{g}}}{\partial \mathrm{t}_{\mathrm{z}}}\right)
\end{aligned}
$$

Since axial equilibrium is satisfied according to the previous section, $\mathbf{f}_{\mathrm{a}, \mathrm{g}}$ is constant and equal to F. Furthermore, since the integration of $\partial \boldsymbol{\varepsilon}_{\mathrm{a}, \mathrm{g}}$ is equal to $\partial \varepsilon_{\mathrm{m}} \mathrm{L}$, as can be verified from (25), and considering the curvature relationships in (23), the above expressions for $\left\langle\mathrm{T}_{\mathrm{y}}, \mathrm{T}_{\mathrm{z}}\right\rangle$ can be simplified to:

$$
\begin{aligned}
& \mathrm{T}_{\mathrm{y}}=\left[\sum_{\mathrm{g}=1}^{\mathrm{n}_{\mathrm{g}}} \mathbf{w}_{\mathrm{g}}\left(\mathbf{m}_{\mathrm{y}, \mathrm{g}} \frac{\partial \mathbf{k}_{\mathrm{y}, \mathrm{g}}}{\partial \mathrm{t}_{\mathrm{y}}}\right)\right]+\mathrm{FL} \frac{\partial \varepsilon_{\mathrm{m}}}{\partial \mathrm{t}_{\mathrm{y}}} \\
& \mathrm{T}_{\mathrm{z}}=\left[\sum_{\mathrm{g}=1}^{\mathrm{n}_{\mathrm{g}}} \mathbf{w}_{\mathrm{g}}\left(\mathbf{m}_{\mathrm{z}, \mathrm{g}} \frac{\partial \mathbf{k}_{\mathrm{z}, \mathrm{g}}}{\partial \mathrm{t}_{\mathrm{z}}}\right)\right]+\mathrm{FL} \frac{\partial \varepsilon_{\mathrm{m}}}{\partial \mathrm{t}_{\mathrm{z}}}
\end{aligned}
$$


where, $\left(\mathbf{m}_{\mathrm{y}, \mathrm{g}}, \mathbf{m}_{\mathrm{z}, \mathrm{g}}\right)$ are established according to Section 5 , the derivatives of $\left(\boldsymbol{\kappa}_{\mathrm{y}, \mathrm{g}}, \boldsymbol{\kappa}_{\mathrm{z}, \mathrm{g}}, \varepsilon_{\mathrm{m}}\right)$ are determined from (23) and (25), and the element axial force F is calculated according to the previous section.

The iterative procedure for calculating $\left\langle\mathrm{t}_{\mathrm{y}}, \mathrm{t}_{\mathrm{z}}\right\rangle$ is continued until the relative magnitude of the increments $\left\langle\Delta \mathrm{t}_{\mathrm{y}}, \Delta \mathrm{t}_{\mathrm{z}}\right\rangle$ becomes very small, as illustrated in Fig. 8.

Upon completion of the iterative procedure, the remaining basic element resistance forces are established from the virtual work equation in a similar manner to $\left\langle\mathrm{T}_{\mathrm{y}}, \mathrm{T}_{\mathrm{z}}\right\rangle$ :

$$
\begin{aligned}
& \mathbf{M}_{\mathrm{y} 1}=\left[\sum_{\mathrm{g}=1}^{\mathrm{n}_{\mathrm{g}}} \mathbf{w}_{\mathrm{g}}\left(\mathbf{m}_{\mathrm{y}, \mathrm{g}} \frac{\partial \mathbf{k}_{\mathrm{y}, \mathrm{g}}}{\partial \theta_{\mathrm{y} 1}}\right)\right]+\mathrm{FL} \frac{\partial \varepsilon_{\mathrm{m}}}{\partial \theta_{\mathrm{y} 1}} \\
& \mathbf{M}_{\mathrm{y} 2}=\left[\sum_{\mathrm{g}=1}^{\mathrm{n}_{\mathrm{g}}} \mathbf{w}_{\mathrm{g}}\left(\mathbf{m}_{\mathrm{y}, \mathrm{g}} \frac{\partial \mathbf{k}_{\mathrm{y}, \mathrm{g}}}{\partial \theta_{\mathrm{y} 2}}\right)\right]+\mathrm{FL} \frac{\partial \varepsilon_{\mathrm{m}}}{\partial \theta_{\mathrm{y} 2}} \\
& \mathbf{M}_{\mathrm{zl}}=\left[\sum_{\mathrm{g}=1}^{\mathrm{n}_{\mathrm{g}}} \mathbf{w}_{\mathrm{g}}\left(\mathbf{m}_{\mathrm{z}, \mathrm{g}} \frac{\partial \mathbf{k}_{\mathrm{z}, \mathrm{g}}}{\partial \theta_{\mathrm{z} 1}}\right)\right]+\mathrm{FL} \frac{\partial \varepsilon_{\mathrm{m}}}{\partial \theta_{\mathrm{z} 1}} \\
& \mathbf{M}_{\mathrm{z} 2}=\left[\sum_{\mathrm{g}=1}^{\mathrm{n}_{\mathrm{g}}} \mathbf{w}_{\mathrm{g}}\left(\mathbf{m}_{\mathrm{z}, \mathrm{g}} \frac{\partial \mathbf{k}_{\mathrm{z}, \mathrm{g}}}{\partial \theta_{\mathrm{z} 2}}\right)\right]+\mathrm{FL} \frac{\partial \varepsilon_{\mathrm{m}}}{\partial \theta_{\mathrm{z} 2}} \\
& \mathbf{M}_{\mathrm{T}}=\frac{\mathrm{GJ}}{\mathrm{L}} \theta_{\mathrm{T}}
\end{aligned}
$$

The presence of an F term in the expressions for the element end moments reflects the ability of the proposed formulation to model the geometrically nonlinear beam-column effect. It is also noted the torsional resistance of the element is based on the linear elastic St. Venant's torsion theory, which does not account for the effect of concrete cracking.

\subsection{Local Tangent Stiffness}

The local tangent stiffness matrix ${ }_{b} \mathbf{k}$ of the quartic formulation is given by:

$$
{ }_{\mathrm{b}} \mathbf{k}_{\mathrm{i}, \mathrm{j}}=\frac{\partial_{\mathrm{c}} \mathbf{f}_{\mathrm{i}}}{\partial_{\mathrm{c}} \mathbf{u}_{\mathrm{j}}} \quad \mathrm{i}=1,8 \quad \& \mathrm{j}=1,8
$$


where ${ }_{\mathrm{c}} \mathbf{u}$ and ${ }_{\mathrm{c}} \mathbf{f}$ are the local freedoms and corresponding resistance forces defined in (21).

Considering (30), (31) and (32), the terms of ${ }_{b} \mathbf{k}$ can be shown to have the following form:

$$
\begin{aligned}
& { }_{b} \mathbf{k}_{i, j}=\left[\sum_{g=1}^{n_{g}} \mathbf{w}_{g}\left(\frac{\partial \mathbf{k}_{y, g}}{\partial_{c} \mathbf{u}_{i}}{ }_{t} \mathbf{k}_{2,2, g} \frac{\partial \mathbf{k}_{y, g}}{\partial_{c} \mathbf{u}_{j}}\right)\right]+{ }_{f} \mathbf{k}_{i, j} \quad \text { for }\left\{\begin{array}{l}
i \\
j
\end{array}\right\}=\left\{\begin{array}{l}
{[1,2,3]} \\
{[1,2,3]}
\end{array}\right\} \\
& { }_{b} \mathbf{k}_{i, j}=\left[\sum_{g=1}^{n_{g}} \mathbf{w}_{g}\left(\frac{\partial \mathbf{k}_{y, g}}{\partial_{c} \mathbf{u}_{i}}{ }_{t} \mathbf{k}_{2,3, g} \frac{\partial \mathbf{k}_{z, g}}{\partial_{c} \mathbf{u}_{j}}\right)\right]+{ }_{\mathrm{f}} \mathbf{k}_{\mathrm{i}, \mathrm{j}} \quad \text { for }\left\{\begin{array}{l}
\mathrm{i} \\
\mathrm{j}
\end{array}\right\}=\left\{\begin{array}{l}
{[1,2,3]} \\
{[4,5,6]}
\end{array}\right\} \\
& { }_{b} \mathbf{k}_{i, j}=\left[\sum_{g=1}^{n_{g}} \mathbf{w}_{g}\left(\frac{\partial \mathbf{k}_{z, g}}{\partial_{c} \mathbf{u}_{i}}{ }_{t} \mathbf{k}_{3,3, g} \frac{\partial \mathbf{k}_{z, g}}{\partial_{c} \mathbf{u}_{j}}\right)\right]+{ }_{f} \mathbf{k}_{i, j} \quad \text { for }\left\{\begin{array}{l}
\mathrm{i} \\
j
\end{array}\right\}=\left\{\begin{array}{l}
{[4,5,6]} \\
{[4,5,6]}
\end{array}\right\} \\
& { }_{b} \mathbf{k}_{i, j}={ }_{b} \mathbf{k}_{j, i} \quad \text { for }\left\{\begin{array}{l}
\mathrm{i} \\
j
\end{array}\right\}=\left\{\begin{array}{l}
{[4,5,6]} \\
{[1,2,3]}
\end{array}\right\} \\
& { }_{b} \mathbf{k}_{i, j}={ }_{b} \mathbf{k}_{j, i}=\frac{\partial F}{\partial_{c} \mathbf{u}_{i}} \quad \text { for }\left\{\begin{array}{l}
i \\
j
\end{array}\right\}=\left\{\begin{array}{c}
{[1,2,3,4,5,6,7]} \\
7
\end{array}\right\} \\
& { }_{\mathrm{b}} \mathbf{k}_{8,8}=\frac{\mathrm{GJ}}{\mathrm{L}}
\end{aligned}
$$

All other terms of ${ }_{b} \mathbf{k}$ are zero

where ${ }_{\mathrm{t}} \mathbf{k}$ is defined in (5) and,

$$
\begin{aligned}
{ }_{\mathrm{f}} \mathbf{k}_{\mathrm{i}, \mathrm{j}}= & \mathrm{L}\left[\left(\frac{\partial \mathrm{F}}{\partial_{\mathrm{c}} \mathbf{u}_{\mathrm{i}}}\right)\left(\frac{\partial \boldsymbol{\varepsilon}_{\mathrm{m}}}{\partial_{\mathrm{c}} \mathbf{u}_{\mathrm{j}}}\right)+\left(\frac{\partial \mathrm{F}}{\partial_{\mathrm{c}} \mathbf{u}_{\mathrm{j}}}\right)\left(\frac{\partial \boldsymbol{\varepsilon}_{\mathrm{m}}}{\partial_{\mathrm{c}} \mathbf{u}_{\mathrm{i}}}\right)+\mathrm{F}\left(\frac{\partial^{2} \boldsymbol{\varepsilon}_{\mathrm{m}}}{\partial_{\mathrm{c}} \mathbf{u}_{\mathrm{i}} \partial_{\mathrm{c}} \mathbf{u}_{\mathrm{j}}}\right)\right] \\
& -\left[\sum_{\mathrm{g}=1}^{\mathrm{n}_{\mathrm{g}}} \mathbf{w}_{\mathrm{g}}\left(\frac{\partial \boldsymbol{\varepsilon}_{\mathrm{a}, \mathrm{g}}}{\partial_{\mathrm{c}} \mathbf{u}_{\mathrm{i}}}{ }_{\mathrm{t}} \mathbf{k}_{1,1, \mathrm{~g}} \frac{\partial \boldsymbol{\varepsilon}_{\mathrm{a}, \mathrm{g}}}{\partial_{\mathrm{c}} \mathbf{u}_{\mathrm{j}}}\right)\right] \quad \text { for }\left\{\begin{array}{l}
\mathrm{i} \\
\mathrm{j}
\end{array}\right\}=\left\{\begin{array}{l}
{[1,2,3,4,5,6]} \\
{[1,2,3,4,5,6]}
\end{array}\right\}
\end{aligned}
$$

All the terms of (33) and (34) can be readily determined from previous expressions, except $\frac{\partial \boldsymbol{\varepsilon}_{\mathrm{a}, \mathrm{g}}}{\partial_{\mathrm{c}} \mathbf{u}_{\mathrm{i}}}$ and $\frac{\partial \mathrm{F}}{\partial_{\mathrm{c}} \mathbf{u}_{\mathrm{i}}}$ which can be derived from the following conditions of incremental axial equilibrium and compatibility: 


$$
\begin{aligned}
& \frac{\partial \mathrm{F}}{\partial_{\mathrm{c}} \mathbf{u}_{\mathrm{i}}}=\frac{\partial \mathbf{f}_{\mathrm{a}, \mathrm{g}}}{\partial_{\mathrm{c}} \mathbf{u}_{\mathrm{i}}}=\frac{\partial \mathbf{f}_{\mathrm{a}, \mathrm{g}+1}}{\partial_{\mathrm{c}} \mathbf{u}_{\mathrm{i}}} \quad \mathrm{g}=1, \mathrm{n}_{\mathrm{g}}-1 \Rightarrow \\
& { }_{\mathrm{t}} \mathbf{k}_{1,1, \mathrm{~g}} \frac{\partial \boldsymbol{\varepsilon}_{\mathrm{a}, \mathrm{g}}}{\partial_{\mathrm{c}} \mathbf{u}_{\mathrm{i}}}+{ }_{\mathrm{t}} \mathbf{k}_{1,2, \mathrm{~g}} \frac{\partial \mathbf{k}_{\mathrm{y}, \mathrm{g}}}{\partial_{\mathrm{c}} \mathbf{u}_{\mathrm{i}}}+{ }_{\mathrm{t}} \mathbf{k}_{1,3, \mathrm{~g}} \frac{\partial \mathbf{k}_{\mathrm{z}, \mathrm{g}}}{\partial_{\mathrm{c}} \mathbf{u}_{\mathrm{i}}}= \\
& { }_{\mathrm{t}} \mathbf{k}_{1,1, \mathrm{~g}+1} \frac{\partial \boldsymbol{\varepsilon}_{\mathrm{a}, \mathrm{g}+1}}{\partial_{\mathrm{c}} \mathbf{u}_{\mathrm{i}}}+{ }_{\mathrm{t}} \mathbf{k}_{1,2, \mathrm{~g}+1} \frac{\partial \mathbf{k}_{\mathrm{y}, \mathrm{g}+1}}{\partial_{\mathrm{c}} \mathbf{u}_{\mathrm{i}}}+{ }_{\mathrm{t}} \mathbf{k}_{1,3, \mathrm{~g}+1} \frac{\partial \mathbf{k}_{\mathrm{z}, \mathrm{g}+1}}{\partial_{\mathrm{c}} \mathbf{u}_{\mathrm{i}}} \quad \mathrm{g}=1, \mathrm{n}_{\mathrm{g}}-1 \\
& \sum_{\mathrm{g}=1}^{\mathrm{n}_{\mathrm{g}}} \mathbf{w}_{\mathrm{g}} \frac{\partial \boldsymbol{\varepsilon}_{\mathrm{a}, \mathrm{g}}}{\partial_{\mathrm{c}} \mathbf{u}_{\mathrm{i}}}=\frac{\partial \varepsilon_{\mathrm{m}}}{\partial_{\mathrm{c}} \mathbf{u}_{\mathrm{i}}} \mathrm{L}
\end{aligned}
$$

leading to:

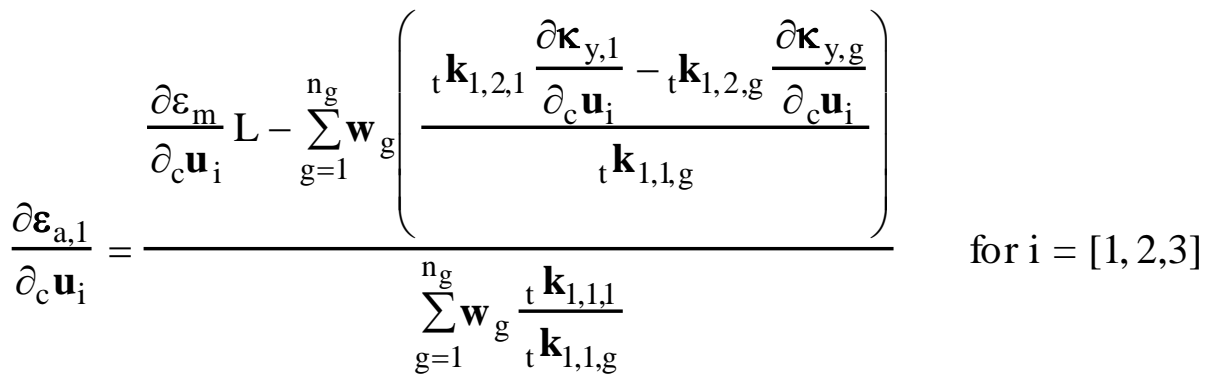

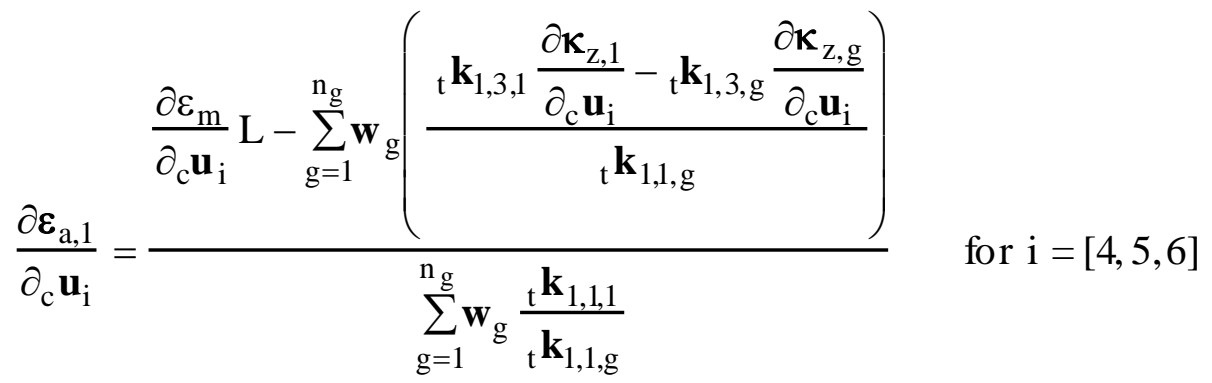

$\frac{\partial \mathrm{F}}{\partial_{\mathrm{c}} \mathbf{u}_{\mathrm{i}}}={ }_{\mathrm{t}} \mathbf{k}_{1,1,1} \frac{\partial \boldsymbol{\varepsilon}_{\mathrm{a}, 1}}{\partial_{\mathrm{c}} \mathbf{u}_{\mathrm{i}}}+{ }_{\mathrm{t}} \mathbf{k}_{1,2,1} \frac{\partial \mathbf{k}_{\mathrm{y}, 1}}{\partial_{\mathrm{c}} \mathbf{u}_{\mathrm{i}}} \quad$ for $\mathrm{i}=[1,2,3]$

$\frac{\partial \mathrm{F}}{\partial_{\mathrm{c}} \mathbf{u}_{\mathrm{i}}}={ }_{\mathrm{t}} \mathbf{k}_{1,1,1} \frac{\partial \boldsymbol{\varepsilon}_{\mathrm{a}, 1}}{\partial_{\mathrm{c}} \mathbf{u}_{\mathrm{i}}}+{ }_{\mathrm{t}} \mathbf{k}_{1,3,1} \frac{\partial \mathbf{k}_{\mathrm{z}, 1}}{\partial_{\mathrm{c}} \mathbf{u}_{\mathrm{i}}} \quad$ for $\mathrm{i}=[4,5,6]$

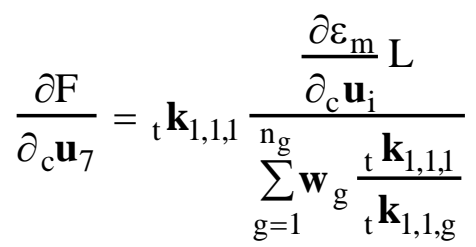

$$
\frac{\partial \boldsymbol{\varepsilon}_{\mathrm{a}, \mathrm{g}}}{\partial_{\mathrm{c}} \mathbf{u}_{\mathrm{i}}}=\frac{\frac{\partial \mathrm{F}}{\partial_{\mathrm{c}} \mathbf{u}_{\mathrm{i}}}-{ }_{\mathrm{t}} \mathbf{k}_{1,2, \mathrm{~g}} \frac{\partial \mathbf{k}_{\mathrm{y}, \mathrm{g}}}{\partial_{\mathrm{c}} \mathbf{u}_{\mathrm{i}}}}{{ }_{\mathrm{t}} \mathbf{k}_{1,1, \mathrm{~g}}} \quad \text { for } \mathrm{i}=[1,2,3]
$$




$$
\frac{\partial \boldsymbol{\varepsilon}_{\mathrm{a}, \mathrm{g}}}{\partial_{\mathrm{c}} \mathbf{u}_{\mathrm{i}}}=\frac{\frac{\partial \mathrm{F}}{\partial_{\mathrm{c}} \mathbf{u}_{\mathrm{i}}}-{ }_{\mathrm{t}} \mathbf{k}_{1,3, \mathrm{~g}} \frac{\partial \mathbf{k}_{\mathrm{z,g}}}{\partial_{\mathrm{c}} \mathbf{u}_{\mathrm{i}}}}{{ }_{\mathrm{t}} \mathbf{k}_{1,1, \mathrm{~g}}} \quad \text { for } \mathrm{i}=[4,5,6]
$$

The $8 \times 8$ tangent stiffness matrix ${ }_{b} \mathbf{k}$ established above must be condensed to the basic $6 \times 6$

matrix ${ }_{c} \mathbf{k}$ to be directly applicable to large displacement incremental analysis using the Eulerian approach (Izzuddin \& Elnashai, 1993-b). The condensation of the two internal freedoms can be performed in accordance with a procedure outlined by Izzuddin (1996).

\subsection{Global Analysis}

The proposed R/C quartic formulation can be utilised in large displacement analysis based on the Eulerian approach (Izzuddin \& Elnashai, 1993-b). This requires three transformations between the element local Eulerian (convected) system and the global reference system common to all elements: (1) transformation of global to local displacements, (2) transformation of local to global resistance forces, and (3) transformation of local to global tangent stiffness matrix for guiding the nonlinear iterative solution procedure. These transformations are presented in detail in (Izzuddin \& Elnashai, 1993-b).

\section{ADAPTIVE NONLINEAR ANALYSIS}

An adaptive nonlinear analysis method was previously developed by Izzuddin (1991) for framed structures, which is based on practical considerations rather than error estimation (Izzuddin, 2000). This method accounts accurately for the effects of geometric and material nonlinearities in static and dynamic analysis, and achieves considerable computational savings, often exceeding $90 \%$, in comparison with the conventional method. The elastic R/C beamcolumn formulation, proposed in this paper, forms one of three main components of the adaptive nonlinear analysis method as applied to 3D R/C frames. The remaining two components are 1) an accurate elasto-plastic formulation of the fibre-type (Izzuddin, 1991; Izzuddin \& Elnashai, 1993-a) capable of modelling the effects of material inelasticity, including steel yielding, concrete compressive softening and general hysteretic behaviour, and 
2) an automatic refinement procedure (Izzuddin \& Lloyd Smith, 2000) governing the transition between the elastic and elasto-plastic elements.

As an overview, adaptive nonlinear analysis of 3D R/C frames is started with one elastic element per member, where each elastic element is checked at the end of an equilibrium step for exceeding its limit of applicability (Izzuddin \& Lloyd Smith, 2000). This limit is generally based on steel yielding and concrete compressive crushing, although a stricter limit can be imposed on concrete compression to control any inaccuracies of the elastic element associated with compressive strain reversal. If an elastic element exceeds its applicability limit in predefined zones over its length, it is refined into a number of elasto-plastic elements in these affected zones, before the nonlinear analysis is resumed from the current equilibrium step. Through the use of adaptive refinement, this procedure enables accurate modelling of the effects of material plasticity, including concrete softening (Zeris \& Mahin, 1988), on the overall member and structural response.

In view of the above, the computational efficiency of adaptive $3 \mathrm{D} R / \mathrm{C}$ frame analysis is maximised on two accounts: (a) on the cross-sectional level, the proposed elastic element is more economical than the elasto-plastic element which involves cross-sectional discretisation into numerous monitoring areas, (b) on the longitudinal element level, one elastic quartic element is capable of accurately modelling a whole $\mathrm{R} / \mathrm{C}$ member in the elastic range, whereas several elasto-plastic elements are required to represent an elasto-plastic member. Thus, by maximising the use of elastic elements and minimising the use of elasto-plastic elements within the structure and during the analysis, considerable computational benefits are achieved without compromising the solution accuracy (Izzuddin \& Lloyd Smith, 2000).

\section{VERIFICATION EXAMPLES}

The proposed quartic formulation has been implemented in the nonlinear analysis program ADAPTIC v2.6.2 (Izzuddin, 1991), which is used herein to verify the formulation accuracy and efficiency. In the two examples presented hereafter, comparison is made between the 
results obtained from one quartic element per member and solutions obtained using ten cubic elements per member. The cubic elements, which are elasto-plastic of the fibre type, have been developed and verified elsewhere (Izzuddin, 1991; Izzuddin \& Elnashai, 1993-a), and results obtained from ten cubic elements per member are considered of sufficient accuracy to provide reference solutions for the validation of the proposed quartic element. However, it should be noted that elastic material properties are assumed for the cubic elements in accordance with Section 3.1, so as to enable the validation of the proposed elastic quartic element. Furthermore, no consideration is given to whether the displacements achieved in the example problems exceed the elastic limit, since one of the aims is to demonstrate the accuracy of the quartic element in accounting for geometric nonlinearities. Of course, the inclusion of the elastic formulation within adaptive elasto-plastic analysis, as discussed in the previous Section, would ensure that the elastic elements do not exceed their elastic limit (Izzuddin \& Lloyd Smith, 2000), although this form of analysis is not considered here.

In all the following example cases, the material properties assumed for the steel reinforcement and concrete are $\left(E_{\mathrm{s}}=200 \times 10^{3} \mathrm{~N} / \mathrm{mm}^{2}, \mathrm{f}_{\mathrm{c}}=20 \mathrm{~N} / \mathrm{mm}^{2}, \varepsilon_{\mathrm{co}}=0.002\right)$. Furthermore, six Gauss integration points are used for each quartic element, unless otherwise stated.

\subsection{Cantilever}

The R/C cantilever, shown in Fig. 9, has a varying reinforcement scheme and is subjected at its tip to concentrated loading applied at the geometric centroid of the rectangular cross-section. The loading consists of an initial axial load $\mathrm{N}$ and a varying transverse load $\mathrm{P}$ making an angle $\alpha$ with the vertical plane. The response of the cantilever to a varying $\mathrm{P}$ at a constant angle $\alpha$ is studied for three values of the initial axial load $(\mathrm{N}=\{0,500,1000\} \mathrm{kN})$. Corresponding to these values, the results obtained using one quartic element are identified by (QN0, QN1, QN2), respectively, whereas the results obtained using ten cubic elements are identified by (CN0, CN1, CN2), respectively. 
For the case of a vertical transverse loading $\left(\alpha=0^{\circ}\right)$, the response of the cantilever at the three levels of axial loading $\mathrm{N}$ to varying $\mathrm{P}$ is shown in Fig. 10. Excellent agreement is observed between the results of one quartic element and ten cubic elements for the three levels of $\mathrm{N}$. The results show that the response at zero $\mathrm{N}$ is virtually linear, corresponding to the cracked response of the cantilever. At the intermediate level of axial load $(\mathrm{N}=500 \mathrm{kN})$, there is an initial vertical displacement due to the asymmetry of the cantilever sections about the horizontal loading plane; the initial response is stiff and corresponds to the uncracked behaviour; and the cracked response is delayed, as expected, until $\mathrm{P}$ is close to the maximum applied value of $50 \mathrm{kN}$. For the highest level of axial load $(\mathrm{N}=1000 \mathrm{kN})$, the initial vertical displacement is even greater, the initial uncracked response is maintained, and the cracked response is delayed even further.

For the case of a horizontal transverse loading $\left(\alpha=90^{\circ}\right)$, the response of the cantilever at the three levels of axial loading $\mathrm{N}$ to varying $\mathrm{P}$ is shown in Fig. 11. Excellent agreement is observed between the results of one quartic element and ten cubic elements for the three levels of $\mathrm{N}$, with slight discrepancies at the maximum applied load $(\mathrm{P}=50 \mathrm{kN})$. This discrepancy is attributed to the difference between the concrete models adopted for the quartic and cubic elements in the post-crushing range - the cubic elements accounting for compressive softening - which is evidenced by the increase in the discrepancy at $(\mathrm{P}=50 \mathrm{kN})$ with an increase in the level of the axial load N. Since the proposed quartic element is only intended to model the elastic response of $\mathrm{R} / \mathrm{C}$ members, these discrepancies are therefore considered irrelevant. It is worth noting that for this case $\left(\alpha=90^{\circ}\right)$, the cracked response for non-zero $\mathrm{N}$ is achieved at lower values of $\mathrm{P}$ in comparison with the previous case $\left(\alpha=0^{\circ}\right)$, which is related to the lower flexural modulus in the horizontal direction. Furthermore, the response becomes even more flexible than the cracked response when $\mathrm{P}$ exceeds $30 \mathrm{kN}$ for $(\mathrm{N}=500 \mathrm{kN})$ or P exceeds 40 $\mathrm{kN}$ for $(\mathrm{N}=1000 \mathrm{kN})$; this is attributed to the geometric nonlinearity introduced by a compressive axial force $\mathrm{N}$, commonly known as the beam-column effect.

The response of the cantilever to a varying transverse load $\mathrm{P}$ applied at different angles $\alpha$ is obtained for an initial axial load $(\mathrm{N}=500 \mathrm{kN})$, with $\mathrm{P}$ varied in five equal steps up to a value 
of $50 \mathrm{kN}$. The results, shown in Fig. 12, depict the variation of the vertical tip displacement with the horizontal tip displacement determined for the five values of load P applied at angles $\left(\alpha=\{0,30,45,60,90\}^{\circ}\right)$. These results demonstrate very good agreement between one quartic element and ten cubic elements, with minor discrepancies at $(\mathrm{P}=50 \mathrm{kN})$ attributed to the same effect discussed previously for the case of horizontal loading $\left(\alpha=90^{\circ}\right)$. It is also observed that while the load-displacement response is nonlinear for all angles $\alpha$, the variation of vertical displacement with horizontal displacement is almost linear for small values of $\alpha$, becoming considerably nonlinear at large values of $\alpha$. As expected, the horizontal displacement for a vertical load $\left(\alpha=0^{\circ}\right)$ remains zero due to symmetry of the cantilever sections about the vertical plane, even after concrete cracking. However, for a horizontal load $\left(\alpha=90^{\circ}\right)$, the symmetry of the elastic modulus about the vertical plane is destroyed, particularly after concrete cracking, which leads to coupling between bending about the vertical and horizontal axes and, consequently, to considerable vertical upwards displacements.

\subsection{Square Frame}

The 3D R/C frame, shown in Fig. 13, has a square plan geometry, with the beams and columns utilising varying and uniform reinforcement schemes, respectively. The frame is considered under the action of varying horizontal concentrated loads $\left(\mathrm{P}_{\mathrm{x}}, \mathrm{P}_{\mathrm{y}}\right)$ - assumed to be components of a horizontal load $\mathrm{P}$ making an angle $\alpha$ with the global $\mathrm{X}$-axis - in the absence/presence of initial vertical loading applied on the columns (Q) and distributed on the beams (w). The response of the frame to a varying $\mathrm{P}$ at a constant angle $\alpha$ is studied with and without initial vertical loading, where the corresponding results from one quartic element per member are identified by (Q0, Q1), respectively, and the corresponding results from ten cubic elements per member are identified by $(\mathbf{C O}, \mathbf{C 1})$, respectively. It is noted that 10 Gauss points are used for the quartic elements representing the frame beams, due to the considerable variation in the reinforcement scheme. 
The response of the frame to a varying $\mathrm{P}$ for $\left(\alpha=0^{\circ}\right)$, that is $\left(\mathrm{P}_{\mathrm{x}}=\mathrm{P}\right)$ and $\left(\mathrm{P}_{\mathrm{y}}=0\right)$, is shown in Figs. 14.a-b for displacements at corner (A) in the global $\mathrm{X}$ and $\mathrm{Z}$ directions, respectively. Excellent agreement is observed between the results of one quartic element and ten cubic elements up to $(\mathrm{P}=140 \mathrm{kN})$ for the case of no vertical loading, and up to $(\mathrm{P}=100 \mathrm{kN})$ for the case of full vertical loading. The slight discrepancy outside these two ranges is again due to differences in the post-crushing response of the concrete models used for the two types of element, which occurs after considerable displacements of $100 \mathrm{~mm}$ in the $\mathrm{X}$ direction. As mentioned previously, since the proposed quartic formulation is only intended to represent the elastic behaviour of R/C members, this discrepancy is considered irrelevant to the stated aim. It is observed from Fig. 14.a that, in the absence of vertical loading, the response of the frame response in the $\mathrm{X}$ direction is slightly nonlinear, which can be attributed to the varying compressive and tensile axial forces in the beams and columns affecting their flexural stiffness. In the presence of vertical loading, the initial frame response in the $\mathrm{X}$ direction is stiffer than for the case of no vertical loading, since cracking in the columns is delayed; however, after considerable cracking, the frame response becomes more flexible, since geometric nonlinearities due to vertical loading become relatively important. The frame response in the $\mathrm{Z}$ direction, shown in Fig. 14.b, indicates, for the case of no vertical loading, an initial upwards movement due to column cracking which is reversed at around $(\mathrm{P}=80 \mathrm{kN})$ due to large $\mathrm{X}$ displacements. For the case of full vertical loading, the upwards movement is delayed until the columns start cracking, with the reversal occurring at around $(\mathrm{P}=60 \mathrm{kN})$.

The response of the frame to a varying $\mathrm{P}$ for $\left(\alpha=45^{\circ}\right)$, that is $\left(\mathrm{P}_{\mathrm{x}}=\mathrm{P}_{\mathrm{y}}=\mathrm{P} / \sqrt{2}\right)$, is shown in Figs. 15.a-b for displacements at corner (A) in the global $\mathrm{X}$ and $\mathrm{Z}$ directions, respectively. Similar observations and comments can be made here as for the previous case $\left(\alpha=0^{\circ}\right)$, demonstrating the ability of the quartic formulation to represent accurately the elastic frame response under general orientation of the loading.

The last point above is further illustrated by considering the variation of the $\mathrm{X}$-displacement with the Y-displacement at corner (A) for four angles $\left(\alpha=\{0,15,30,45\}^{\circ}\right)$, with $\mathrm{P}$ varied up to $200 \mathrm{kN}$ in five equal steps. The results, shown in Figs. 16 and 17 for the two cases of zero 
and full vertical loading, respectively, demonstrate the ability of the quartic formulation to model the elastic frame response up to considerable displacements of around $100 \mathrm{~mm}$, beyond which concrete crushing is initiated. It is noted that while the load-displacement response for the frame is evidently nonlinear, the variation of the $\mathrm{X}$ displacement with the $\mathrm{Y}$ displacement is almost linear for all angles $\alpha$, indicating the constraining nature of the square frame geometry.

The modelling efficiency of the proposed quartic formulation is evident, since only one element per member is required to model the geometrically nonlinear behaviour of R/C beamcolumns in the elastic range. More significantly, the computational superiority of the quartic element can be illustrated in terms of CPU demand; on a Silicon Graphics Indy workstation with $32 \mathrm{Mb}$ of memory, the average CPU demand of the frame analyses using the quartic formulation is $6.4 \mathrm{sec}$, whereas the average CPU demand using the cubic formulation is 76 sec. This represents a computational saving by the proposed quartic formulation of more than $90 \%$ for this type of problem.

\section{CONCLUSIONS}

This paper presents a new elastic beam-column formulation for $\mathrm{R} / \mathrm{C}$ members of $3 \mathrm{D}$ framed structures. The proposed formulation is intended to model the geometrically nonlinear elastic response of $\mathrm{R} / \mathrm{C}$ beam-columns using only one element per member. This is an essential requirement for nonlinear adaptive analysis, where analysis is always started with one elastic element per member, and automatic mesh refinement into more computationally demanding elasto-plastic elements is performed when and where necessary, during analysis and within the structure, respectively.

The new formulation is derived in a local Eulerian system, where quartic shape functions are used for the two transverse displacements, hence the name 'quartic formulation'. No shape functions are employed for the axial displacement; instead, the more effective constant axial force criterion is utilised, which is deemed responsible for the accuracy of the quartic formulation, avoiding the overstiff response associated with conventional displacement-based 
finite element formulations. A linear elastic model is adopted for steel, whereas a piecewise relationship is adopted for concrete which neglects tensile stresses and assumes a parabolicconstant response in the compressive range. While compressive concrete softening is not accounted for, a constant response being assumed in the post-crushing range to ensure stability of the internal element iterative procedures, this is deemed irrelevant to the present formulation which is intended to represent only the nonlinear elastic response. The generalised cross-sectional response is established for general $\mathrm{R} / \mathrm{C}$ cross-sections through decomposition into coarse rectangular areas, and a novel approach, based on integration over triangular subdomains, is proposed for determining the contribution of rectangular areas to the overall cross-section response. The paper presents details of transforming the generalised response of cross-sections to an overall response of the element, covering the satisfaction of the constant axial force criterion and the static condensation of internal element freedoms.

Two examples demonstrate the accuracy of the quartic formulation in modelling the nonlinear elastic response of a whole $\mathrm{R} / \mathrm{C}$ member with a varying reinforcement scheme using only one element. Slight discrepancies from the results of ten cubic elements at high levels of loading are attributed to the post-crushing softening response of concrete modelled by the cubic formulation, which is outside the scope of the present elastic formulation. The modelling advantages of the quartic element are pointed out, and, more significantly, its ability to achieve computational savings of more than $90 \%$ in comparison with an adequate mesh of cubic elements is highlighted.

Finally, it is worth reiterating that the proposed elastic quartic formulation provides one of three main components of nonlinear adaptive elasto-plastic analysis of 3D R/C frames. The remaining components, which have already been developed, are an elasto-plastic cubic formulation (Izzuddin \& Elnashai, 1993-a) and applicability criteria which establish whether the elastic limit is exceeded in the concrete or the steel reinforcement of a $\mathrm{R} / \mathrm{C}$ member represented by an elastic quartic element (Izzuddin \& Lloyd Smith, 2000). These criteria are used to guide the automatic mesh refinement process of an elastic quartic element into the more computationally demanding elasto-plastic cubic elements, when and where necessary. 
Recent work by the authors (Izzuddin \& Lloyd Smith, 2000) has shown that the overall adaptive elasto-plastic method for $3 \mathrm{D} \mathrm{R} / \mathrm{C}$ frames is capable of achieving computational savings exceeding $90 \%$ in comparison with conventional nonlinear analysis without a consequential loss of accuracy, an important advantage which is made possible by the accuracy and efficiency of the elastic quartic formulation presented in this paper. 


\section{NOTATION}

$\mathrm{a}, \mathrm{b}$ :

$a_{c}, b_{c}:$

$a_{p}, b_{p}:$

$\mathbf{A}_{\mathrm{s}, \mathrm{i}}$ :

$\mathrm{E}_{\mathrm{s}}$ :

$\mathrm{f}_{\mathrm{a}}, \mathbf{f}_{\mathrm{ac}, \mathrm{i}}, \mathrm{f}_{\mathrm{as}}:$

$\mathbf{f}_{\mathrm{a}, \mathrm{g}}$ :

$\mathrm{f}_{\mathrm{ac}}^{\mathrm{m}}, \mathrm{f}_{\mathrm{ac} 1}^{\mathrm{m}}, \mathrm{f}_{\mathrm{ac} 2}^{\mathrm{m}}$ :

c f :

GJ :

$\mathrm{k}$ :

${ }_{\mathrm{b}} \mathbf{k}$ :

f $\mathbf{k}:$

${ }_{\mathrm{t}} \mathbf{k},{ }_{\mathrm{t}} \mathbf{k}_{\mathrm{c}, \mathrm{i}},{ }_{\mathrm{t}} \mathbf{k}_{\mathrm{s}}$ : dimensions of rectangular concrete block in local y and $\mathrm{z}$ directions.

integration boundaries for concrete block defined in (11).

integration boundaries for concrete block.

cross-sectional area of steel bar (i).

elastic Young's modulus of steel reinforcement.

axial forces of overall section, concrete block (i), and steel reinforcement.

$f_{a}$ at Gauss point $(g)$.

axial force of concrete block and contributions from integrating $\sigma_{c l}$ and $\sigma_{c 2}$ with reference to maximal corner (m).

compressive strength of concrete.

local forces of quartic element $\left.{ }^{\prime} \mathrm{M}_{\mathrm{y} 1}, \mathrm{M}_{\mathrm{y} 2}, \mathrm{~T}_{\mathrm{y}}, \mathrm{M}_{\mathrm{z} 1}, \mathrm{M}_{\mathrm{z} 2}, \mathrm{~T}_{\mathrm{z}}, \mathrm{F}, \mathrm{M}_{\mathrm{T}}\right\rangle^{\mathrm{T}}$.

St. Venant's torsional rigidity.

confinement factor for concrete.

local element tangent stiffness matrix before static condensation of internal freedoms.

local element tangent stiffness associated with axial force.

generalised tangent stiffness matrices of overall section, concrete block (i), and steel reinforcement. 
${ }_{\mathrm{t}} \mathbf{k}_{\mathrm{c}}^{\mathrm{m}}$ :

L:

m, n, q:

$\mathrm{m}_{\mathrm{y}}, \mathbf{m}_{\mathrm{yc}, \mathrm{i}}, \mathrm{m}_{\mathrm{ys}}$

$\mathbf{m}_{\mathrm{y}, \mathrm{g}}$ :

$\mathrm{m}_{\mathrm{yc}}^{\mathrm{m}}, \mathrm{m}_{\mathrm{yc} 1}^{\mathrm{m}}, \mathrm{m}_{\mathrm{yc} 2}^{\mathrm{m}}$ from integrating $\sigma_{c 1}$ and $\sigma_{c 2}$, referred to maximal corner (m).

$\mathrm{m}_{\mathrm{z}}, \mathbf{m}_{\mathrm{zc}, \mathrm{i}}, \mathrm{m}_{\mathrm{zs}}$ : bending moments in local $\mathrm{z}$ direction of overall section, concrete block (i), and steel reinforcement.

$\mathbf{m}_{\mathrm{z}, \mathrm{g}}$ :

$\mathrm{m}_{\mathrm{zc}}^{\mathrm{m}}, \mathrm{m}_{\mathrm{zc} 1}^{\mathrm{m}}, \mathrm{m}_{\mathrm{zc} 2}^{\mathrm{m}}$ :

$\mathrm{n}_{\mathrm{c}}, \mathrm{n}_{\mathrm{s}}, \mathrm{n}_{\mathrm{g}}$ :

$r_{p}, s_{p}:$

$\mathbf{T}_{\mathrm{m}}:$

$\mathrm{T}_{\mathrm{yeq}}, \mathrm{T}_{\mathrm{zeq}}$ :

generalised tangent stiffness matrix of concrete block with reference to maximal corner (m).

element length.

identifiers for a concrete block of the maximal corner, adjacent corner in the local y direction, and adjacent corner in the local $\mathrm{z}$ direction.

bending moments in local y direction of overall section, concrete block (i), and steel reinforcement.

$\mathrm{m}_{\mathrm{y}}$ at Gauss point $(\mathrm{g})$.

bending moments in local y direction of concrete block and contributions $\mathrm{m}_{\mathrm{z}}$ at Gauss point $(\mathrm{g})$.

bending moments in local $\mathrm{z}$ direction of concrete block and contributions from integrating $\sigma_{c 1}$ and $\sigma_{c 2}$, referred to maximal corner (m).

number of rectangular concrete blocks and steel bars over cross-section, and number of Gauss points over element length.

concrete block ratios defined in (9).

concrete block ratios defined in (15).

transformation matrix for concrete block from maximal corner $(\mathrm{m})$ to cross-section reference origin.

equivalent internal element loads due to distributed loading. 
c $\mathbf{u}$ : local freedoms of quartic element $\left\{\theta_{\mathrm{y} 1}, \theta_{\mathrm{y} 2}, \mathrm{t}_{\mathrm{y}}, \theta_{\mathrm{zl}}, \theta_{\mathrm{z} 2}, \mathrm{t}_{\mathrm{z}}, \Delta, \theta_{\mathrm{T}}\right\rangle^{\mathrm{T}}$.

$\mathrm{v}(\mathrm{x})$ : $\quad$ displacement of reference line in local y direction.

$\mathrm{w}(\mathrm{x})$ : $\quad$ displacement of reference line in local $\mathrm{z}$ direction.

$\mathbf{w}_{\mathrm{g}}$ : $\quad$ integration weighting factor for Gauss point $(\mathrm{g})$.

$\mathrm{x}, \mathrm{y}, \mathrm{z}$ : local coordinates for quartic element.

$\mathrm{y}_{\mathrm{c}}^{\mathrm{o}}, \mathrm{z}_{\mathrm{c}}^{\mathrm{o}}$ : $\quad$ local $\mathrm{y}$ and $\mathrm{z}$ coordinates of a concrete block centre.

$\mathbf{y}_{\mathrm{s}, \mathrm{i}}, \mathbf{z}_{\mathrm{s}, \mathrm{i}}$ : local $\mathrm{y}$ and $\mathrm{z}$ coordinates of steel bar (i).

$\varepsilon_{\mathrm{a}}, \kappa_{\mathrm{y}}, \kappa_{\mathrm{z}}: \quad$ centroidal axial strain and curvatures in local y and $\mathrm{z}$ directions.

$\boldsymbol{\varepsilon}_{\mathrm{a}, \mathrm{g}}, \boldsymbol{\kappa}_{\mathrm{y}, \mathrm{g}}, \boldsymbol{\kappa}_{\mathrm{z}, \mathrm{g}}: \quad$ relevant terms for Gauss point $(\mathrm{g})$.

$\varepsilon_{\mathrm{a}}^{\mathrm{m}}, \kappa_{\mathrm{y}}^{\mathrm{m}}, \kappa_{\mathrm{z}}^{\mathrm{m}}: \quad$ centroidal axial strain and curvatures in local y and $\mathrm{z}$ directions, referred to maximal concrete block corner (m).

$\varepsilon_{\mathrm{c}}, \boldsymbol{\varepsilon}_{\mathrm{c}, \mathrm{i}}: \quad$ strain in concrete, and strain at corner (i) of a concrete block.

$\varepsilon_{\mathrm{co}}: \quad$ compressive crushing strain of concrete.

$\varepsilon_{\mathrm{m}}: \quad$ average centroidal axial strain over element length.

$\varepsilon_{\mathrm{p}}, \varepsilon_{\mathrm{p}, \mathrm{i}}: \quad$ compressive strain increment of concrete beyond $\varepsilon_{\mathrm{co}}$ at any point and at corner (i) of concrete block.

$\varepsilon_{\mathrm{s}}, \boldsymbol{\varepsilon}_{\mathrm{s}, \mathrm{i}}: \quad$ strain in steel, and strain in steel bar (i).

$\sigma_{\mathrm{c}}: \quad$ stress in concrete.

$\sigma_{c 1}, \sigma_{c 2}: \quad$ piecewise stress functions for concrete, defined in (2).

$\sigma_{\mathrm{s}}: \quad$ stress in steel reinforcement. 
$\xi_{\mathrm{i}}, \eta_{\mathrm{i}}$ : natural coordinates of corner (i) of concrete block, defined in (8.b).

\section{REFERENCES}

1. Chen, W.F. and Saleeb, A.F., 1994. Constitutive Equations for Engineering Materials. Vol. 2: Plasticity and Modelling. 2nd edition. Elsevier, Amsterdam.

2. El-Metwally, S. E., El-Shahhat, A. M. and Chen, W. F., 1990. "3-D Nonlinear Analysis of R/C Slender Columns”, Computers \& Structures, Vol. 37, No. 5, pp. 863-872.

3. Izzuddin, B.A., Karayannis, C.G. and Elnashai, A.S., 1994. "Advanced Nonlinear Formulation for Reinforced Concrete Beam-Columns”, Journal of Structural Engineering, ASCE, Vol. 120, No. 10, pp. 2913-2934.

4. Izzuddin, B.A. and Elnashai, A.S., 1993-a. "Adaptive Space Frame Analysis: Part II, A Distributed Plasticity Approach", Structures and Buildings Journal, Proceedings of the Institution of Civil Engineers, Vol. 99, No. 3, pp. 317-326.

5. Izzuddin, B.A. and Elnashai, A.S., 1993-b. "Eulerian Formulation for Large Displacement Analysis of Space Frames", Journal of Engineering Mechanics, ASCE, Vol. 119, No. 3, pp. 549-569.

6. Izzuddin, B.A., 1996. "Quartic Formulation for Elastic Beam-Columns Subject to Thermal Effects”, Journal of Engineering Mechanics, ASCE, Vol. 122, No. 9, pp. 861-871.

7. Izzuddin, B.A. and Lloyd Smith, D., 2000. "Efficient Nonlinear Analysis of Elasto-Plastic 3D R/C Frames Using Adaptive Techniques”, Computers \& Structures, Vol. 78, pp. 549573.

8. Izzuddin, B.A., 1991. "Nonlinear Dynamic Analysis of Framed Structures", PhD Thesis, Department of Civil Engineering, Imperial College, London. 
9. Izzuddin, B.A., 2000. "Adaptivity in Nonlinear Structural Analysis of Frames: Perspectives of Practicality and Accuracy", Computational Methods for Shell and Spatial Structures, (IASS-IACM’00), M. Papadrakakis et al. (eds.).

10. Karayannis, C.G., Izzuddin, B.A. and Elnashai, A.S., 1994. "Application of Adaptive Analysis to Reinforced Concrete Frames", Journal of Structural Engineering, ASCE, Vol. 120, No. 10, pp. 2935-2957.

11. Morgan, V. A., 1971. Biaxial Bending Simplified. V. A. Morgan, Middlesex.

12. Rasheed H.A.S. and Dinno, K. S., 1994. "An Efficient Nonlinear Analysis of RC Sections”, Computers \& Structures, Vol. 53, No. 3, pp. 613-623.

13. Sfakianakis, M.G. and Fardis, M.N., 1991. "Bounding Surface Model for Cyclic Bending of RC Sections", Journal of Engineering Mechanics, ASCE, Vol. 117, No. 12, pp. 27482769.

14. Spacone, E., Ciampi, V. and Filippou, F. C., 1996. "Mixed Formulation Of Nonlinear Beam Finite Element”, Computers \& Structures, Vol. 58, No. 1, pp. 71-83.

15. Spacone, E. and Filippou, F. C., 1994. "R/C Beam-Column Element for Nonlinear Frame Analysis", in Analysis \& Computation, Franklin Y. Cheng (ed.), pp. 155-164.

16. Vidosa, F. G., Kotsovos, M. D. and Pavlovic, M. N., 1991. "Nonlinear Finite-Element Analysis of Concrete Structures: Performance of a Fully Three-Dimensional Brittle Model", Computers \& Structures, Vol. 40, No. 5, pp. 1287-1306.

17. Zeris, C.A. and Mahin, S.A., 1988. "Analysis of Reinforced Concrete Beam-Columns under Uniaxial Excitation”, Journal of Structural Engineering, ASCE, Vol. 114, No. 4, pp. 804-820. 


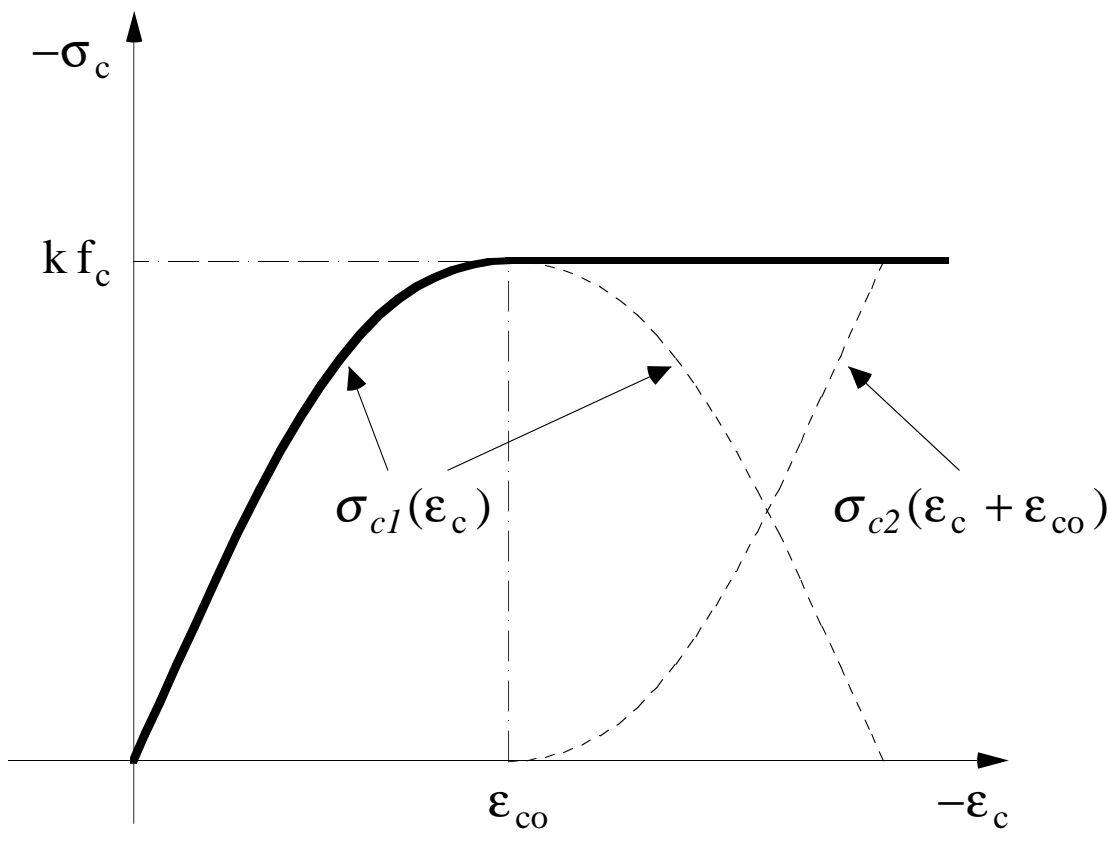

Figure 1. Concrete stress-strain model 


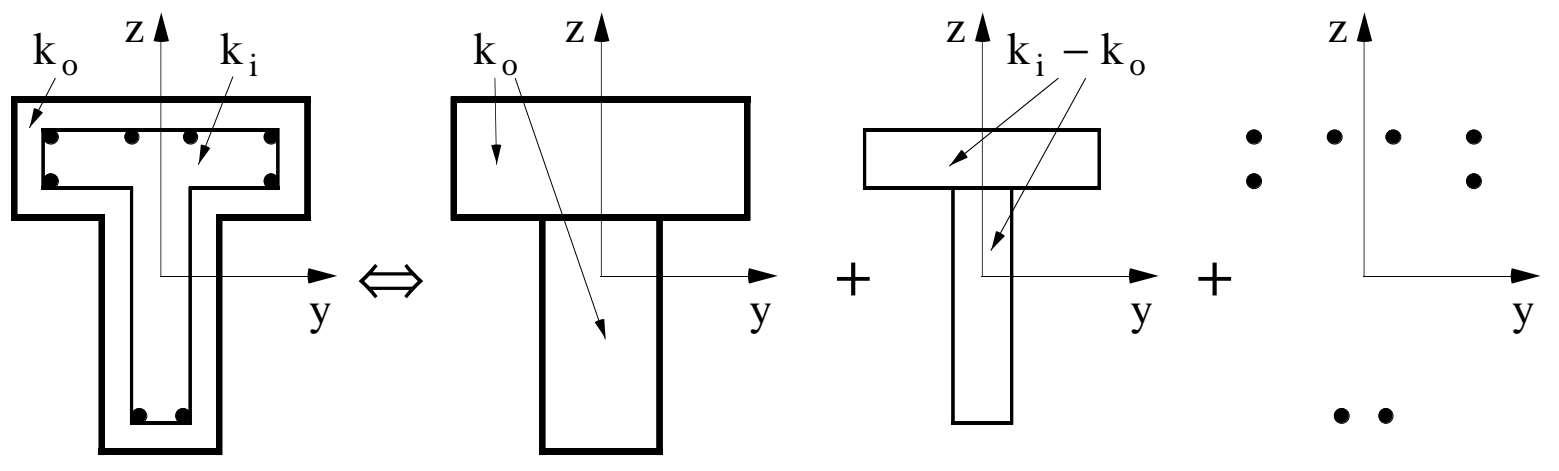

Figure 2. Representation of R/C T-section by rectangular areas 


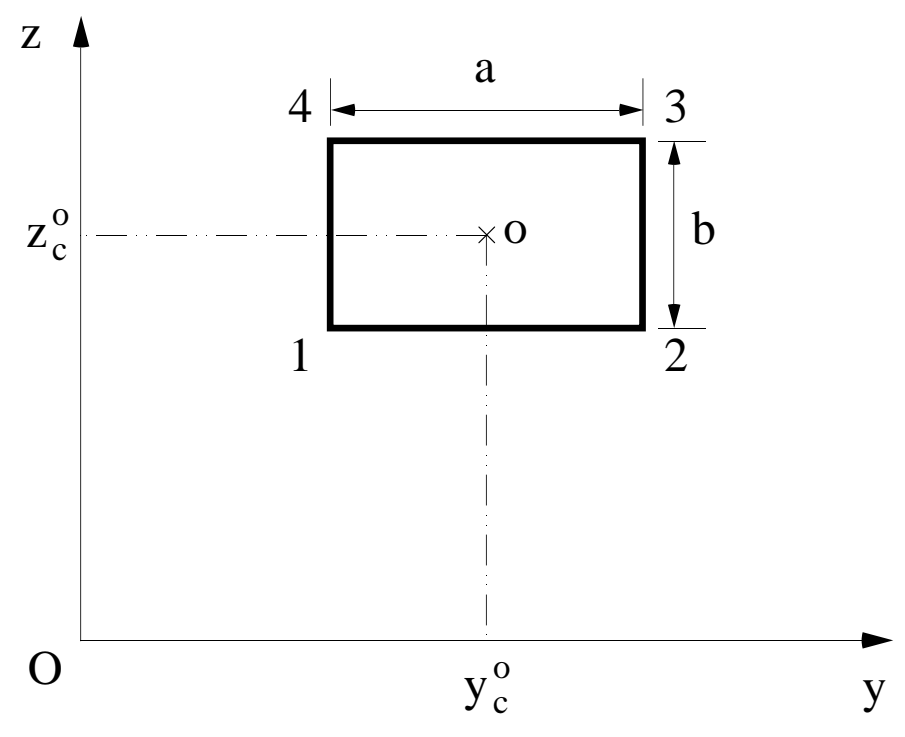

Figure 3. Typical rectangular concrete block 

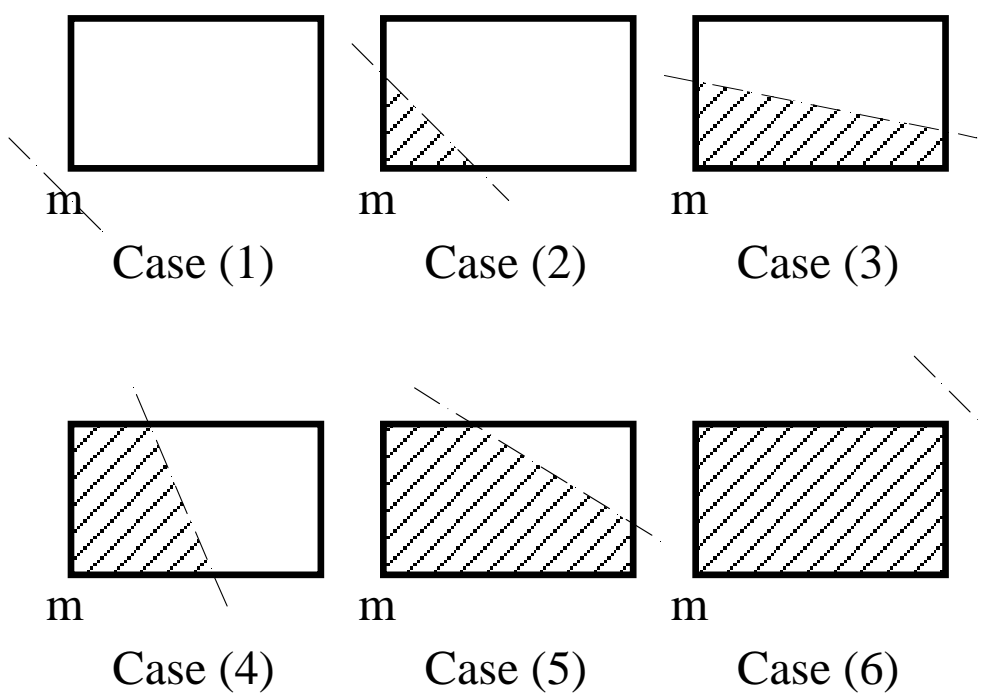

Figure 4. Integration domains over concrete block 


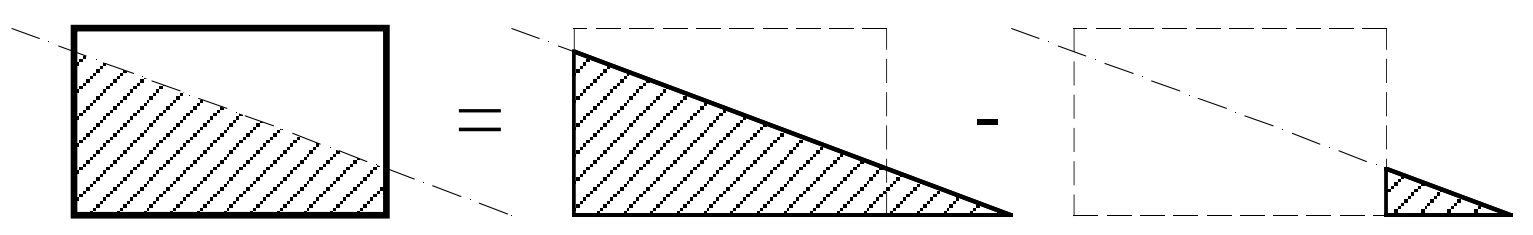

Figure 5. Decomposition of case (3) domain into two triangular domains 

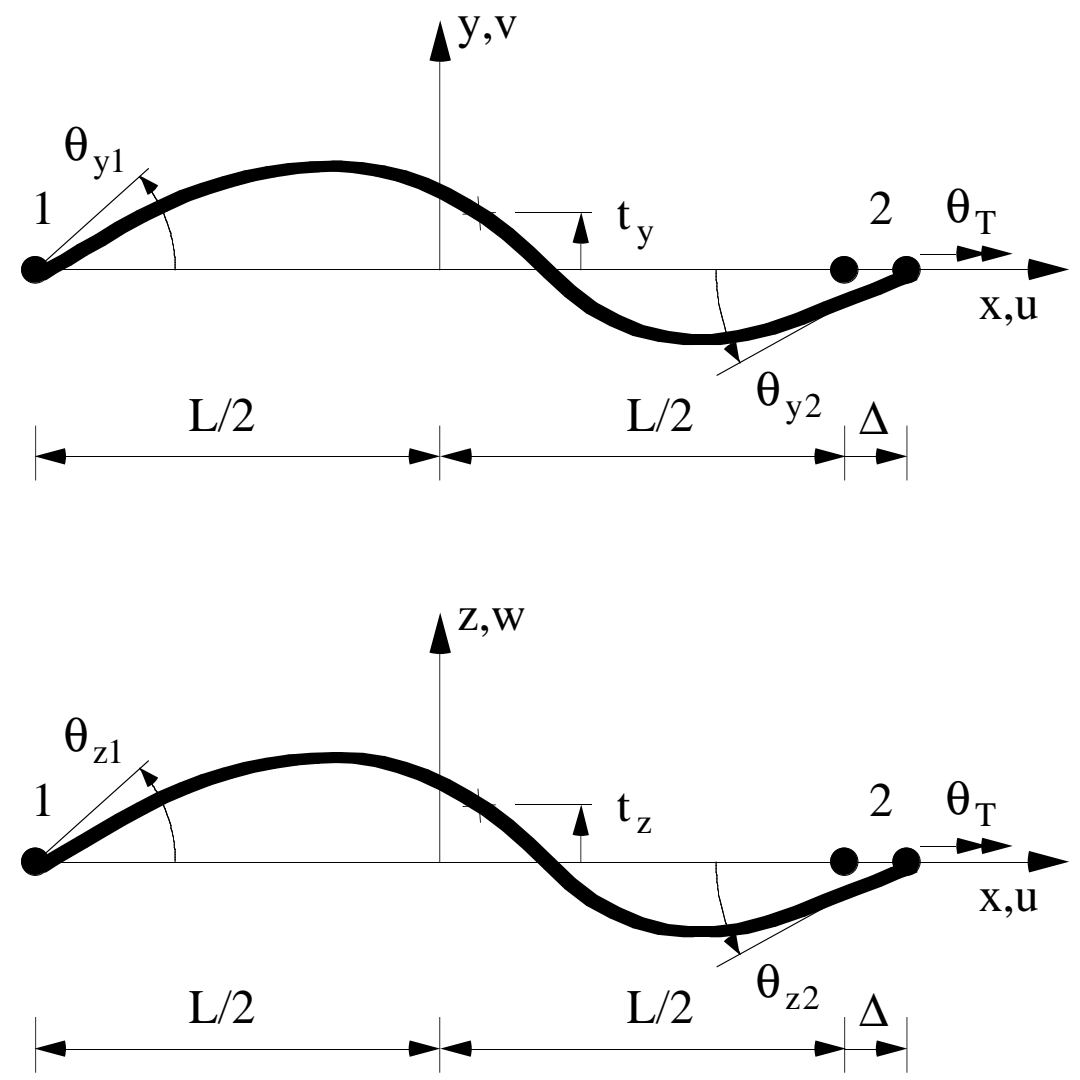

Figure 6. Local freedoms of quartic formulation 


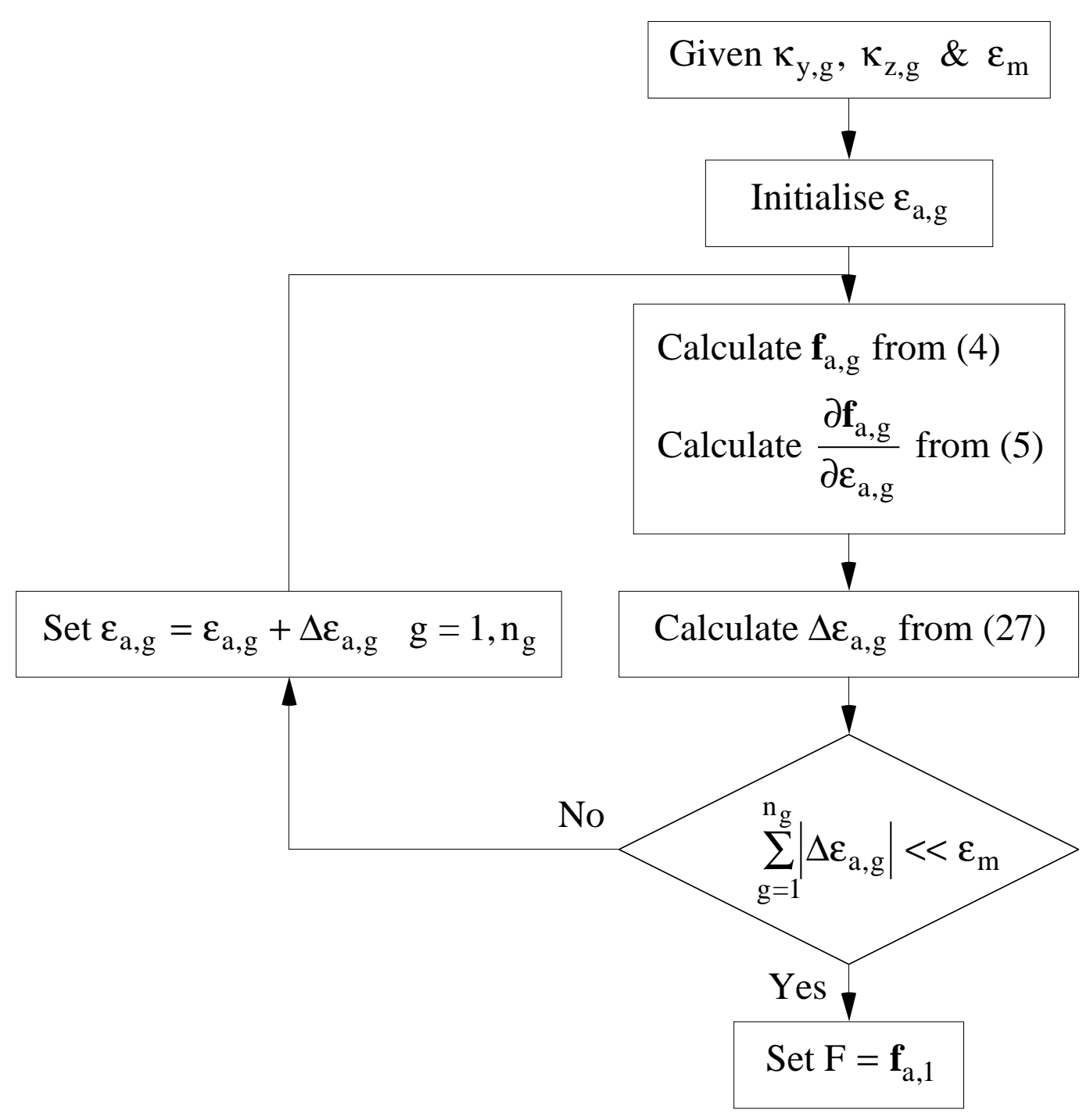

Figure 7. Iterative procedure for axial equilibrium 


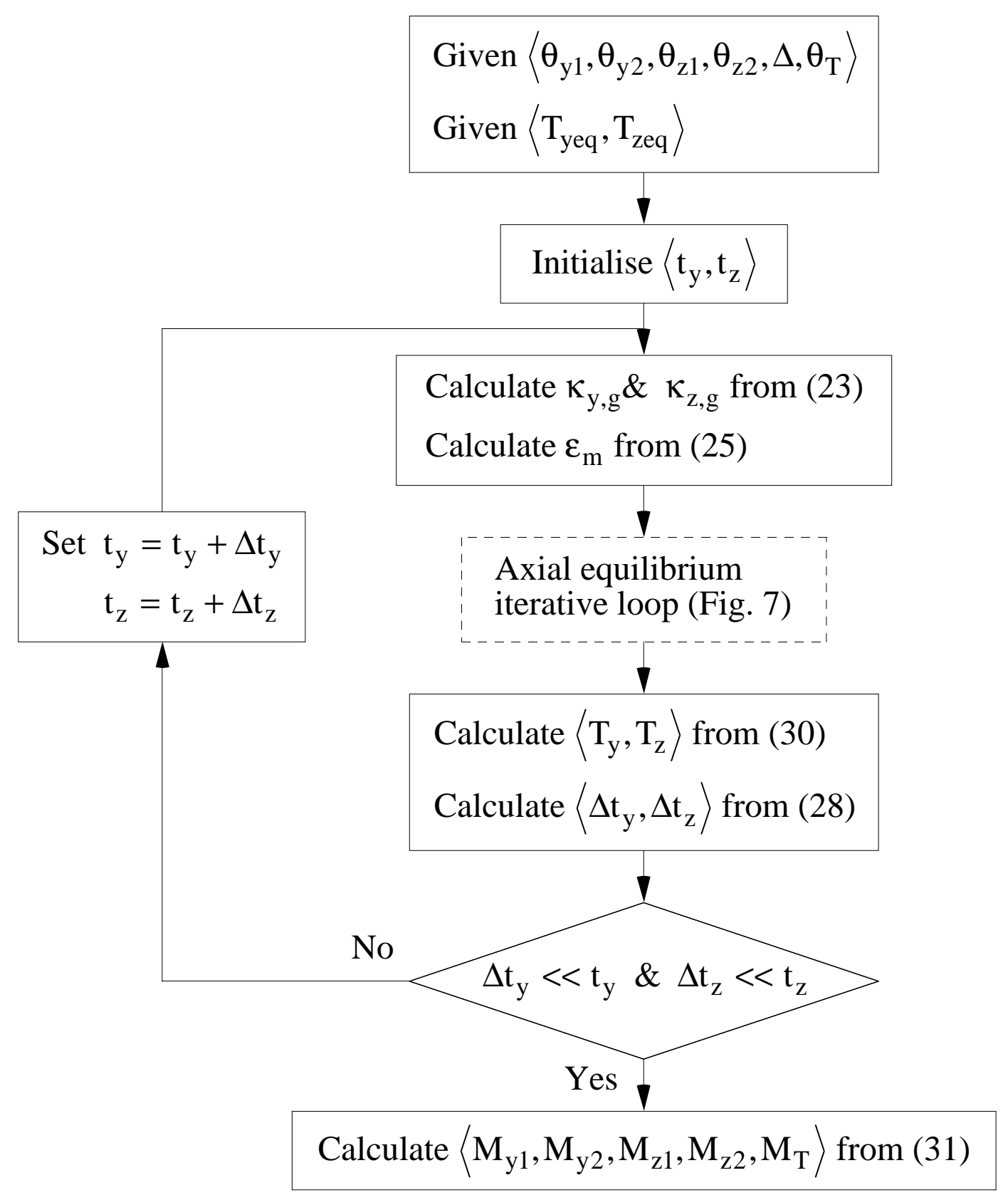

Figure 8. Iterative procedure for internal freedoms 

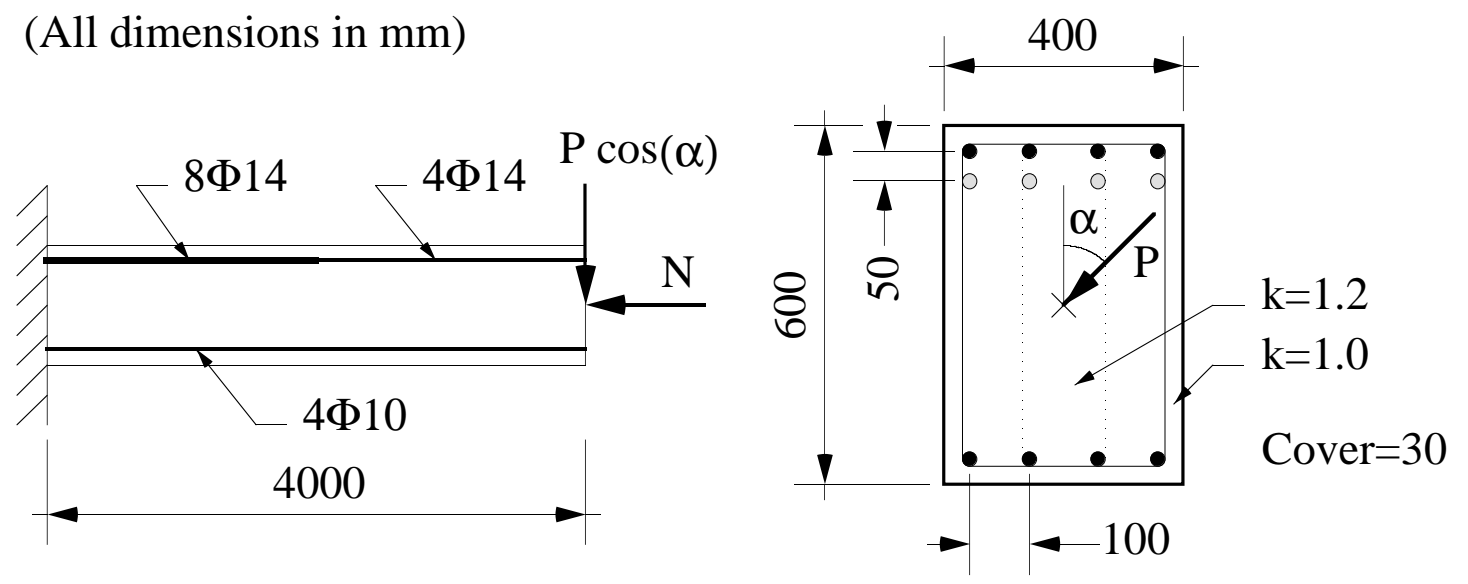

Figure 9. Geometric configuration and loading of R/C cantilever 


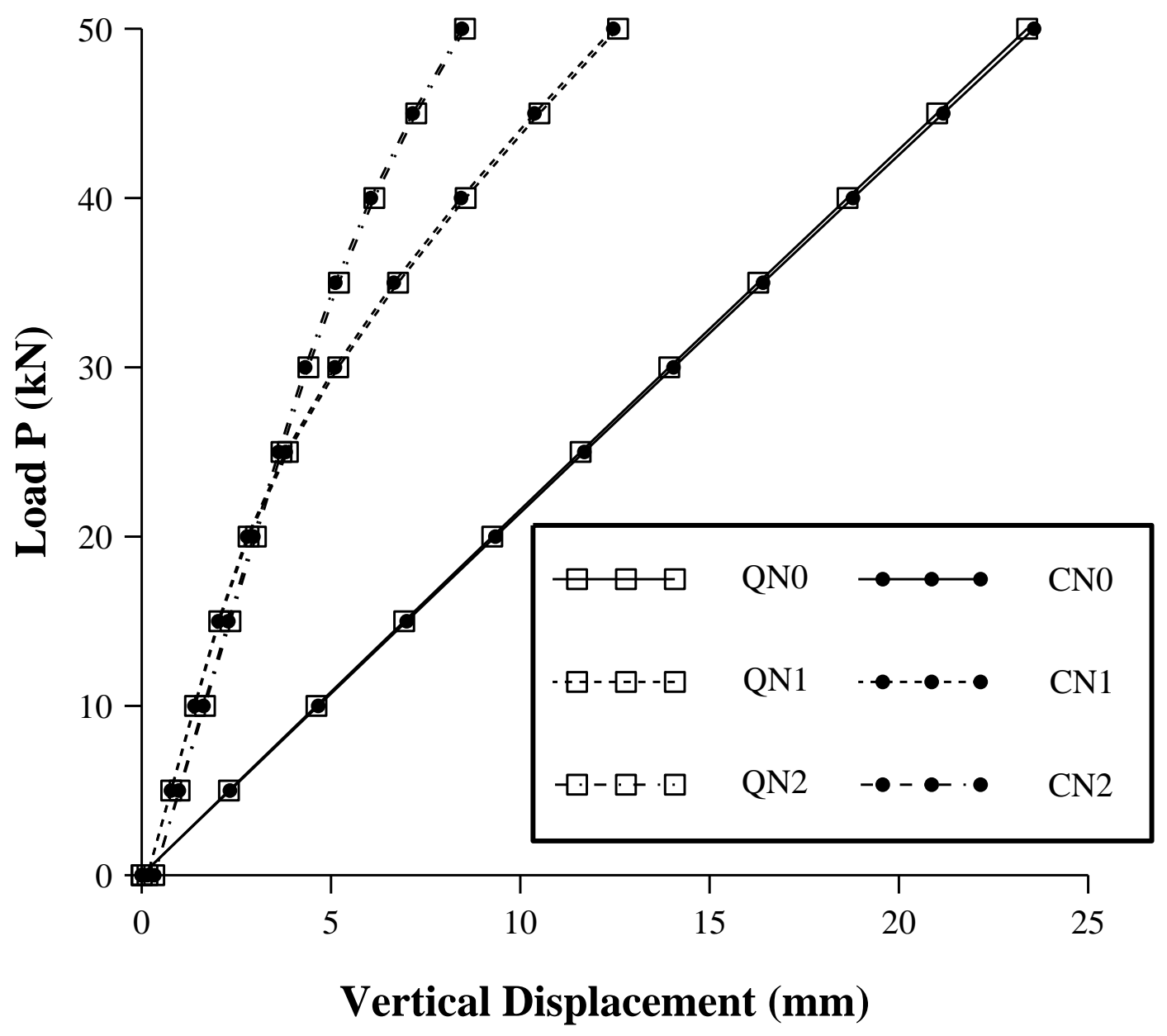

Figure 10. Response of cantilever to vertical load $\left(\alpha=0^{\circ}\right)$ 


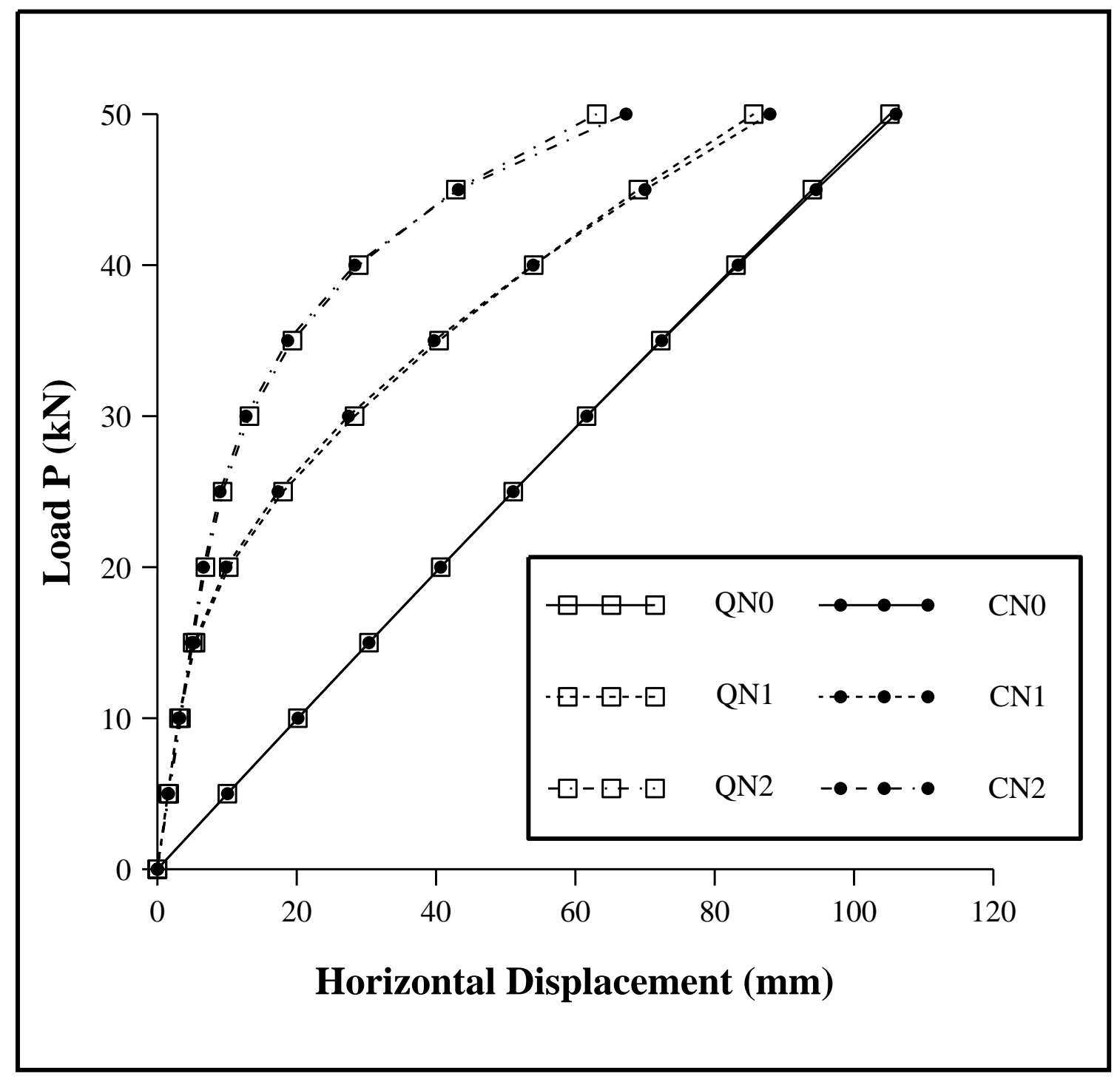

Figure 11. Response of cantilever to horizontal load $\left(\alpha=90^{\circ}\right)$ 


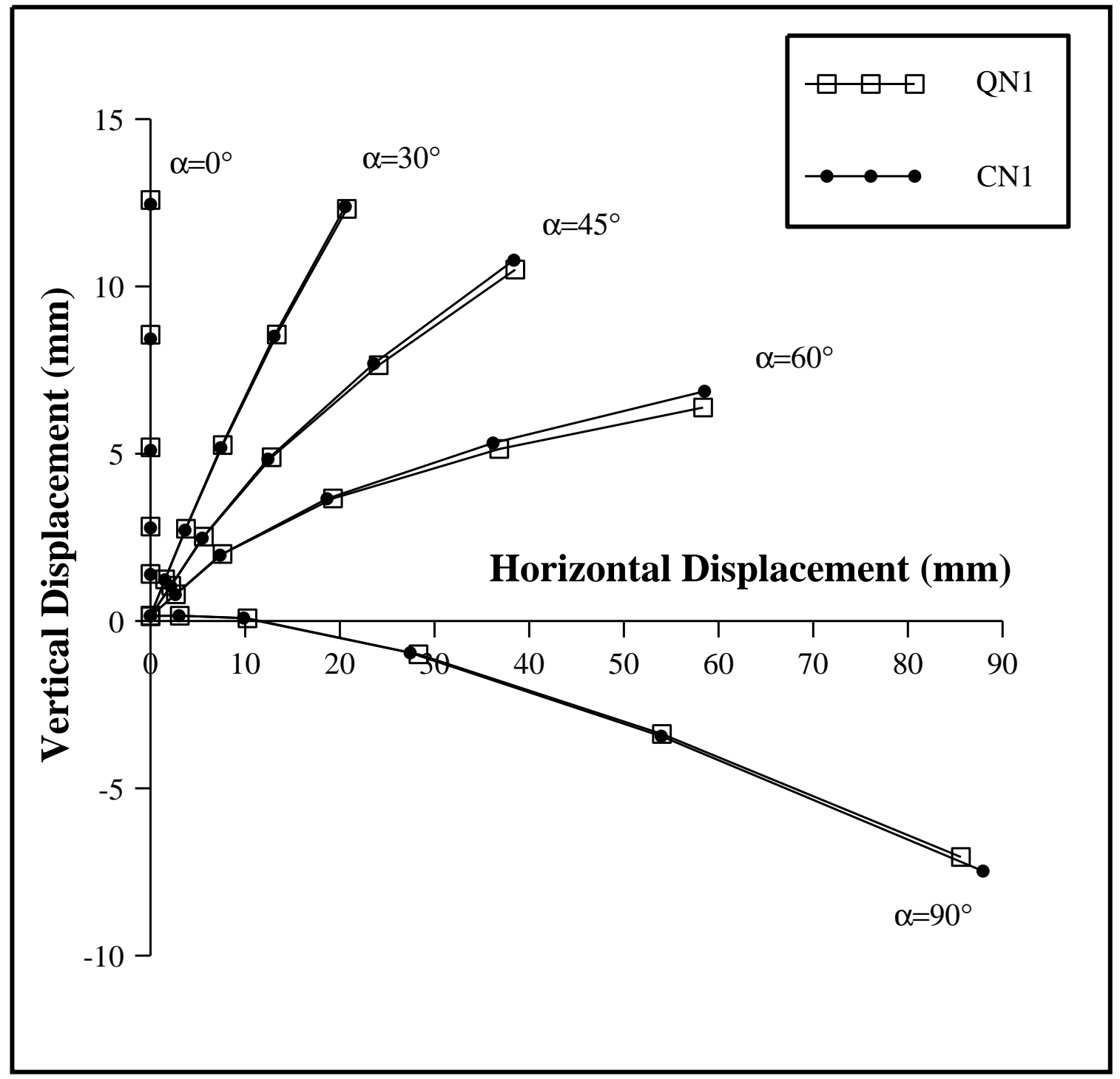

Figure 12. Response of cantilever to inclined transverse load for $(\mathrm{N}=500 \mathrm{kN})$ 


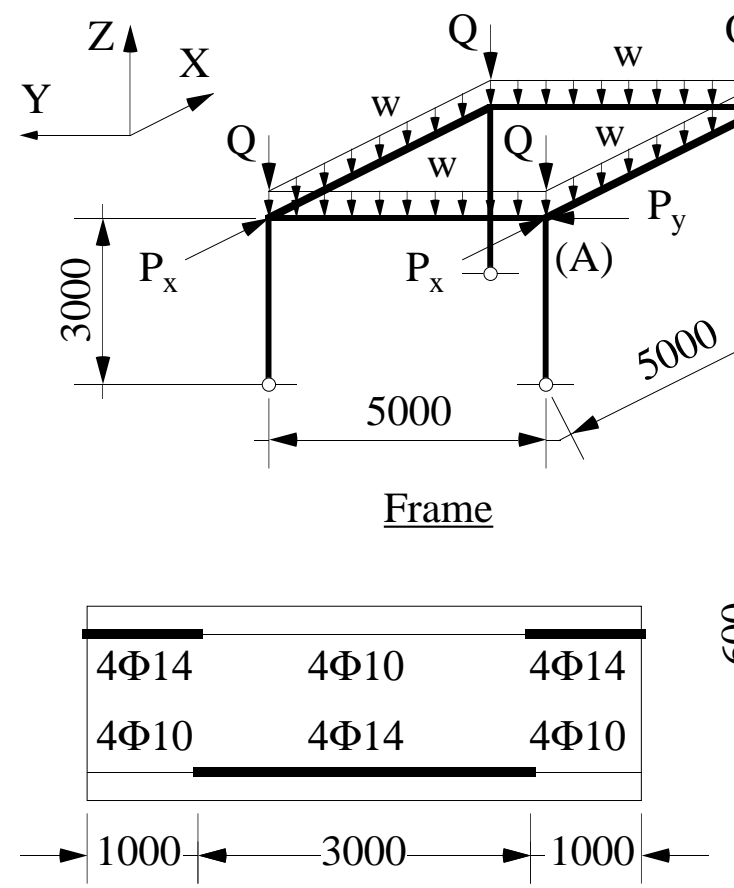

$\underline{\text { Beam Reinforcement }}$

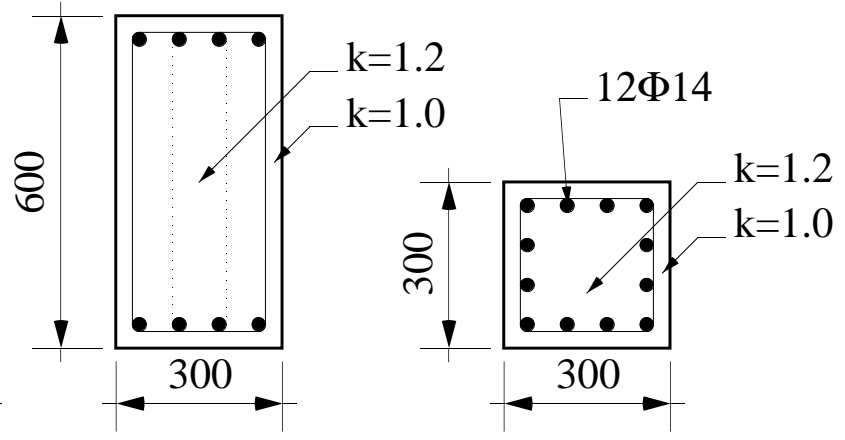

$\underline{\text { Beam Section }}$
(All dimensions in $\mathrm{mm}$ )

$\mathrm{P}_{\mathrm{x}}=\mathrm{P} \cos (\alpha), \quad \mathrm{P}_{\mathrm{y}}=\mathrm{P} \sin (\alpha)$

$\mathrm{Q}=200 \mathrm{kN}, \quad \mathrm{w}=10 \mathrm{kN} / \mathrm{m}$

Cover $=30$

Bar spacing $=80$

Figure 13. Geometric configuration and loading of R/C square frame 


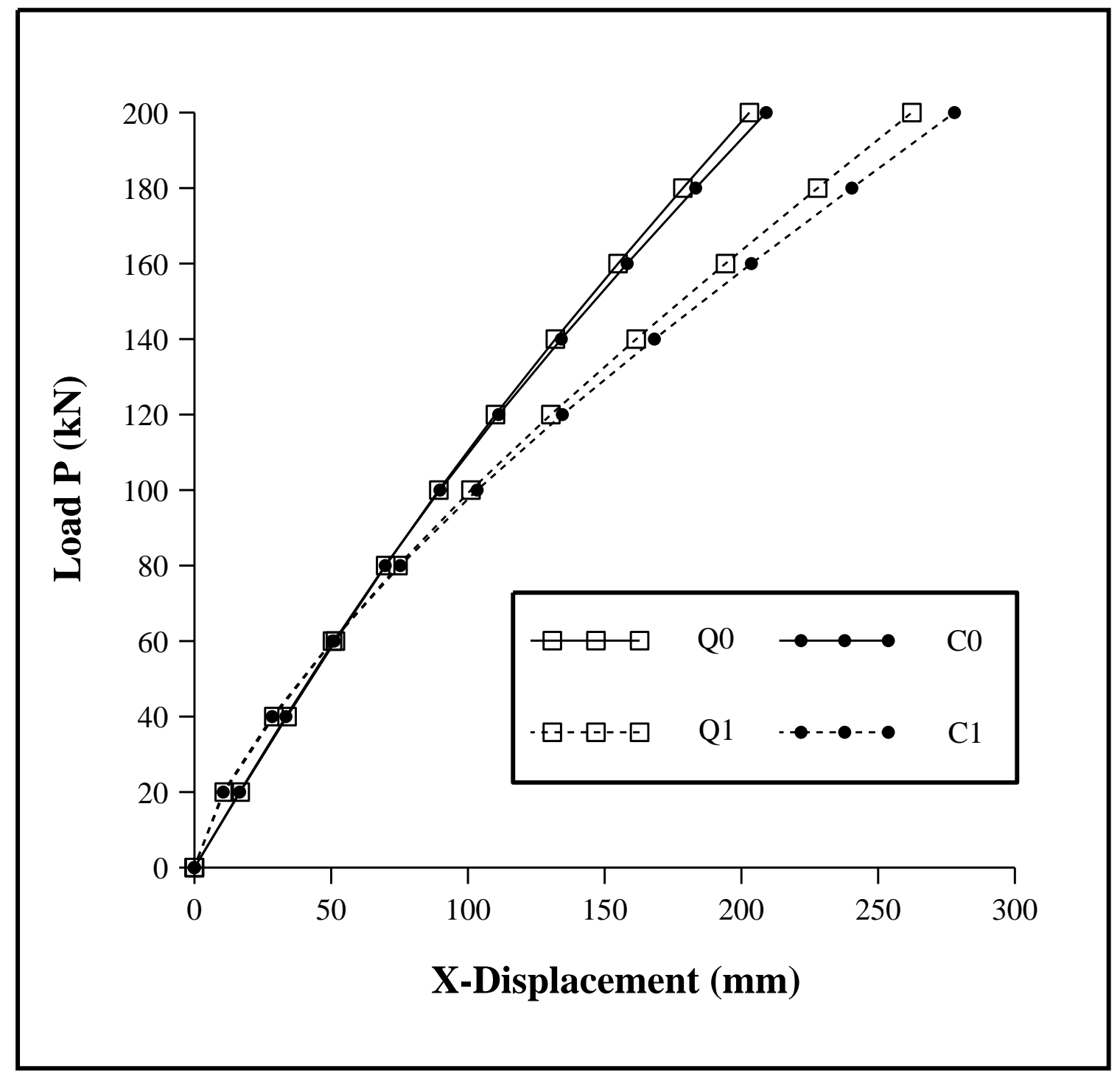

Figure 14.a. Response of square frame at (A) in $X$ direction for $\left(\alpha=0^{\circ}\right)$ 


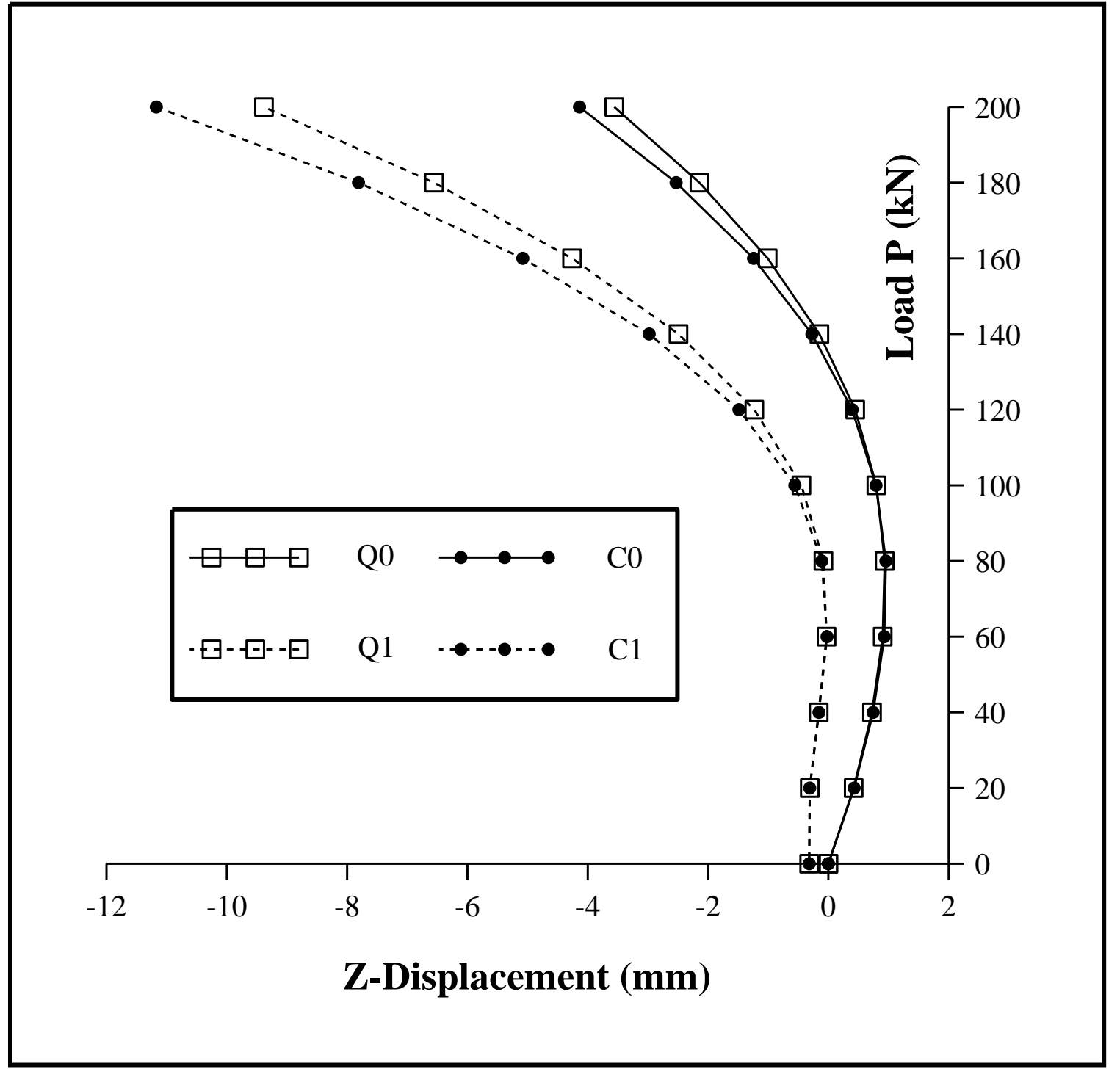

Figure 14.b. Response of square frame at (A) in $\mathrm{Z}$ direction for $\left(\alpha=0^{\circ}\right)$ 


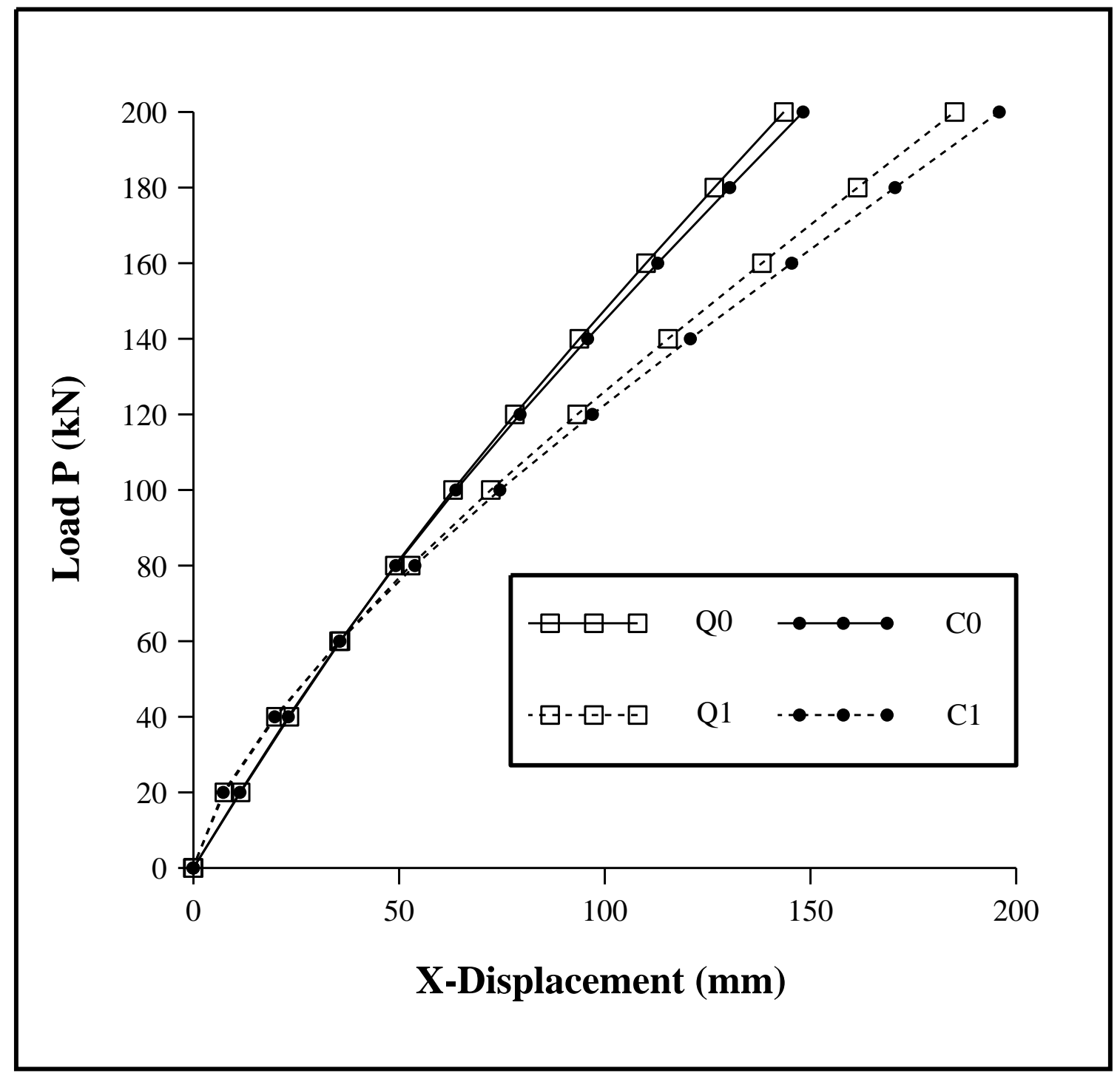

Figure 15.a. Response of square frame at (A) in $X$ direction for $\left(\alpha=45^{\circ}\right)$ 


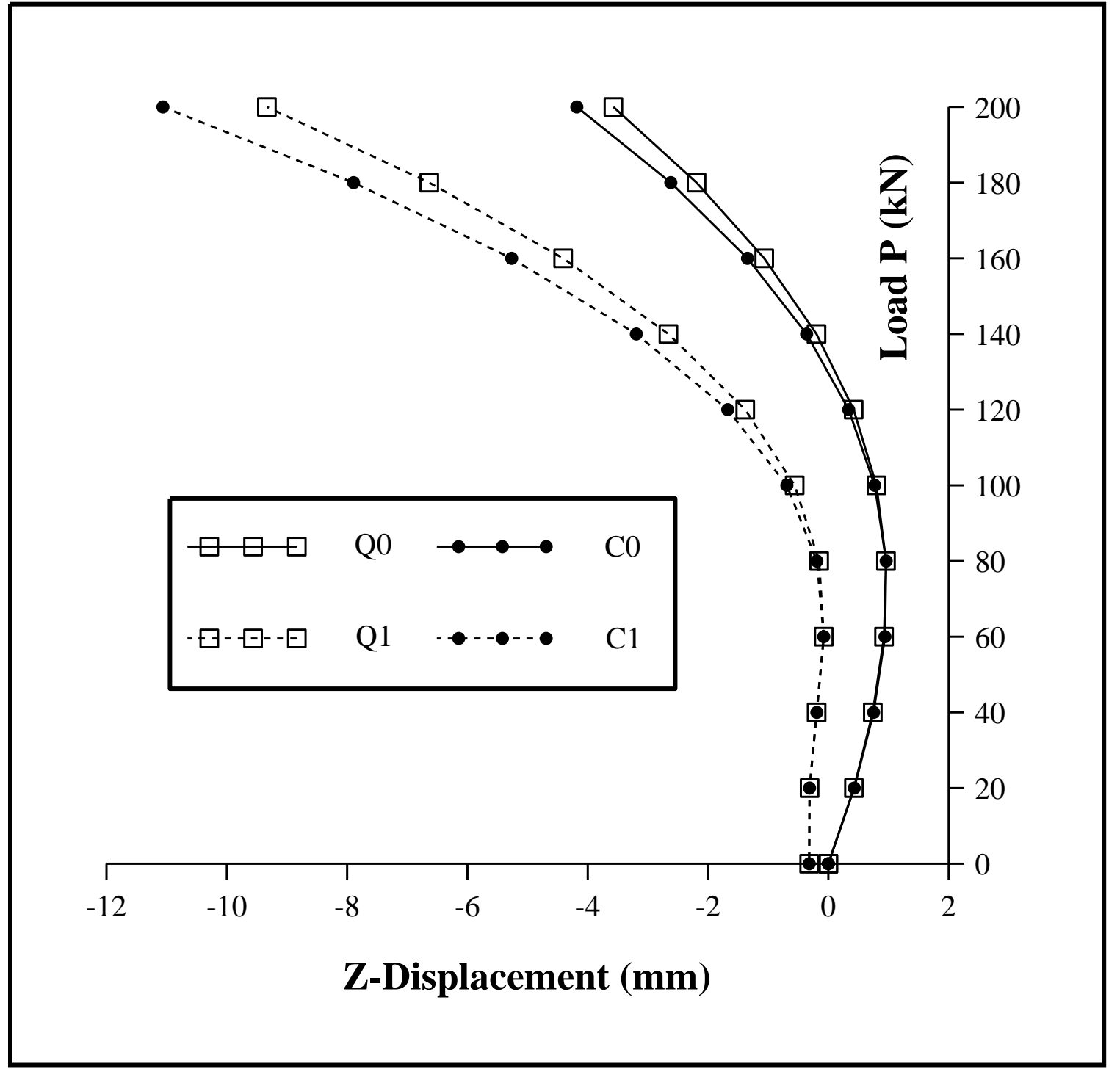

Figure 15.b. Response of square frame at (A) in $\mathrm{Z}$ direction for $\left(\alpha=45^{\circ}\right)$ 


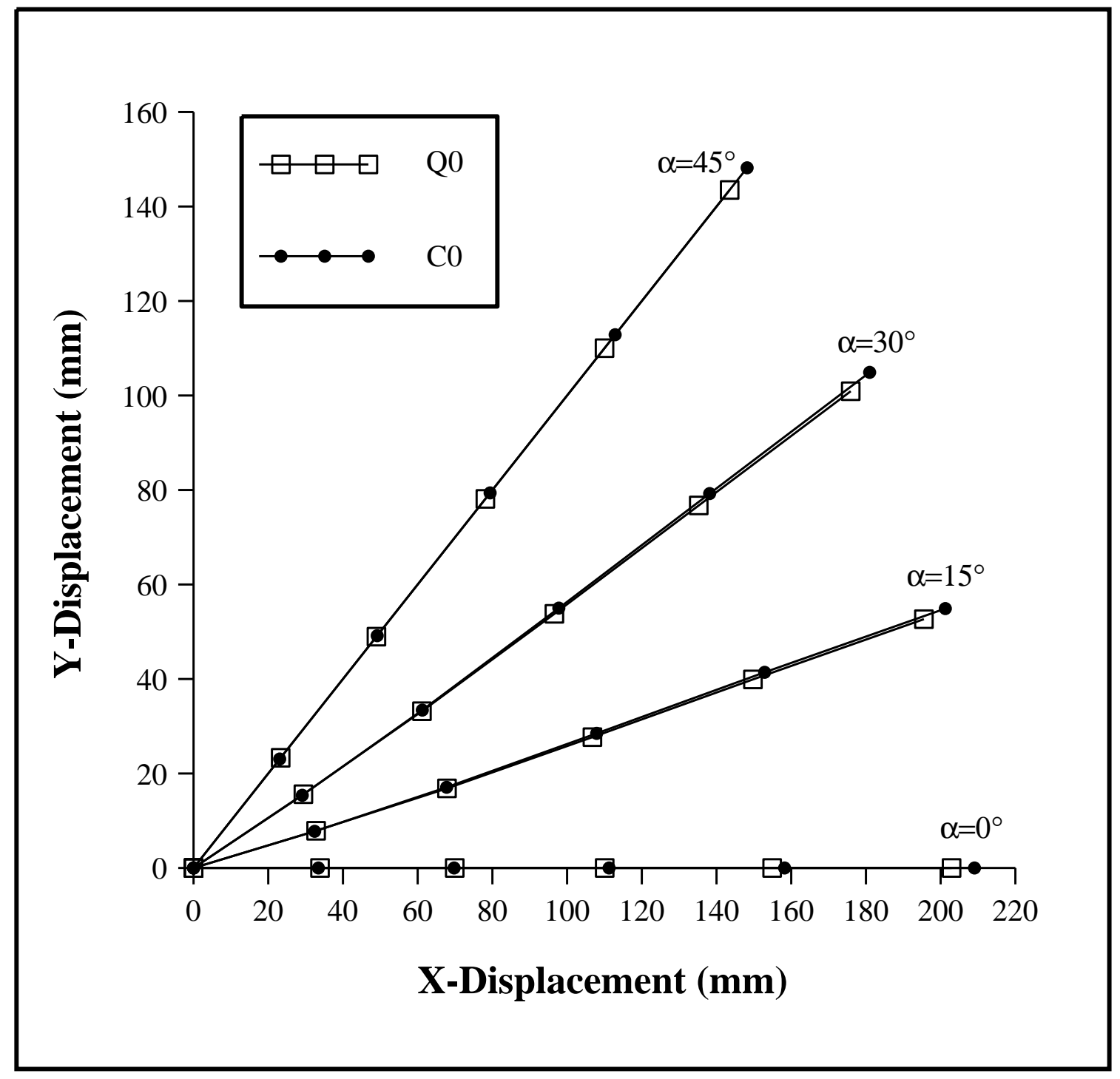

Figure 16. Response of square frame at (A): no vertical loading 


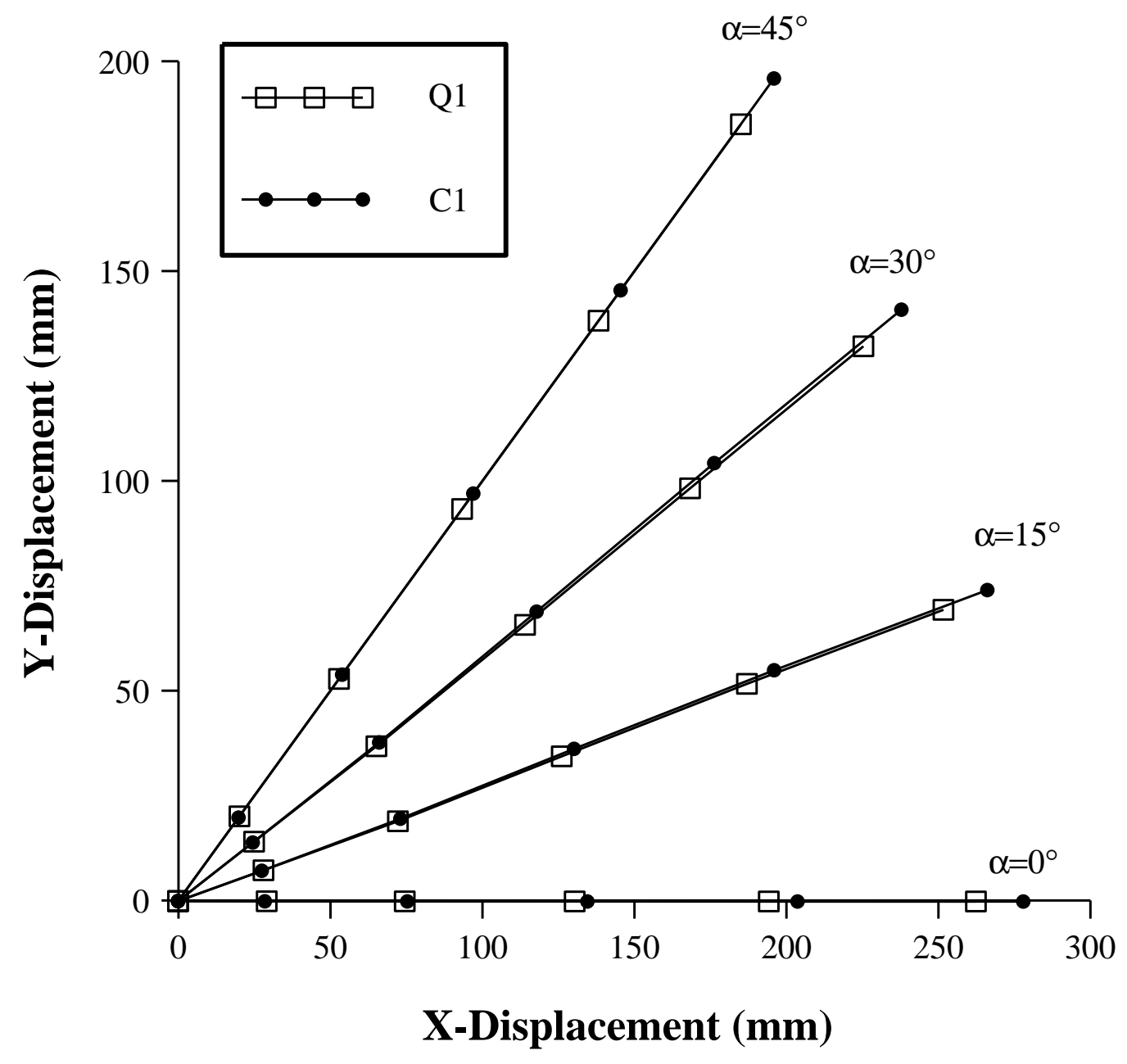

Figure 17. Response of square frame at (A): full vertical loading 